\title{
Computing Eigenvalues of Discontinuous Sturm-Liouville Problems with Eigenparameter in All Boundary Conditions Using Hermite Approximation
}

\author{
M. M. Tharwat, ${ }^{1,2}$ A. H. Bhrawy, ${ }^{1,2}$ and A. S. Alofi ${ }^{1}$ \\ ${ }^{1}$ Department of Mathematics, Faculty of Science, King Abdulaziz University, Jeddah 21589, Saudi Arabia \\ ${ }^{2}$ Department of Mathematics, Faculty of Science, Beni-Suef University, Beni-Suef 62511, Egypt \\ Correspondence should be addressed to A. H. Bhrawy; alibhrawy@yahoo.co.uk
}

Received 23 March 2013; Revised 24 July 2013; Accepted 28 July 2013

Academic Editor: Jose L. Gracia

Copyright (c) 2013 M. M. Tharwat et al. This is an open access article distributed under the Creative Commons Attribution License, which permits unrestricted use, distribution, and reproduction in any medium, provided the original work is properly cited.

The eigenvalues of discontinuous Sturm-Liouville problems which contain an eigenparameter appearing linearly in two boundary conditions and an internal point of discontinuity are computed using the derivative sampling theorem and Hermite interpolations methods. We use recently derived estimates for the truncation and amplitude errors to investigate the error analysis of the proposed methods for computing the eigenvalues of discontinuous Sturm-Liouville problems. Numerical results indicating the high accuracy and effectiveness of these algorithms are presented. Moreover, it is shown that the proposed methods are significantly more accurate than those based on the classical sinc method.

\section{Introduction}

The mathematical modeling of many practical problems in mechanics and other areas of mathematical physics requires solutions of boundary value problems (see, for instance, [1-7]). It is well known that many topics in mathematical physics require the investigation of the eigenvalues and eigenfunctions of Sturm-Liouville-type boundary value problems. The literature on computing eigenvalues of various types of Sturm-Liouville problems is little and we refer to [8-15].

Sampling theory is one of the most powerful results in signal analysis. It is of great need in signal processing to reconstruct (recover) a signal (function) from its values at a discrete sequence of points (samples). If this aim is achieved, then an analog (continuous) signal can be transformed into a digital (discrete) one and then it can be recovered by the receiver. If the signal is band-limited, the sampling process can be done via the celebrated Whittaker, Shannon, and Kotel'nikov (WSK) sampling theorem [16-18]. By a bandlimited signal with band width $\sigma, \sigma>0$, that is, the signal contains no frequencies higher than $\sigma / 2 \pi$ cycles per second (cps), we mean a function in the Paley-Wiener space $B_{\sigma}^{2}$ of the entire functions of the exponential type at most $\sigma$ which are
$L^{2}(\mathbb{R})$-functions when restricted to $\mathbb{R}$. Assume that $f(t) \in$ $B_{\sigma}^{2} \subset B_{2 \sigma}^{2}$. Then $f(t)$ can be reconstructed via the Hermitetype sampling series

$f(t)$

$$
=\sum_{n=-\infty}^{\infty}\left[f\left(\frac{n \pi}{\sigma}\right) S_{n}^{2}(t)+f^{\prime}\left(\frac{n \pi}{\sigma}\right) \frac{\sin (\sigma t-n \pi)}{\sigma} S_{n}(t)\right]
$$

where $S_{n}(t)$ is the sequences of sinc functions

$$
S_{n}(t):= \begin{cases}\frac{\sin (\sigma t-n \pi)}{(\sigma t-n \pi)}, & t \neq \frac{n \pi}{\sigma}, \\ 1, & t=\frac{n \pi}{\sigma} .\end{cases}
$$

Series (1) converges absolutely and uniformly on $\mathbb{R}$ (cf. [1922]). Sometimes, series (1) is called the derivative sampling 
theorem. Our task is to use formula (1) to compute the eigenvalues numerically of differential equation

$$
\begin{array}{r}
-\mathfrak{y}^{\prime \prime}(x, \mu)+q(x) \mathfrak{y}(x, \mu)=\mu^{2} \mathfrak{y}(x, \mu), \\
x \in[-1,0) \cup(0,1],
\end{array}
$$

with boundary conditions

$$
\begin{gathered}
\mathscr{L}_{1}(\mathfrak{y}):=\left(\alpha_{1}^{\prime} \mu^{2}-\alpha_{1}\right) \mathfrak{y}(-1, \mu) \\
-\left(\alpha_{2}^{\prime} \mu^{2}-\alpha_{2}\right) \mathfrak{y}^{\prime}(-1, \mu)=0 \\
\mathscr{L}_{2}(\mathfrak{y}):=\left(\beta_{1}^{\prime} \mu^{2}+\beta_{1}\right) \mathfrak{y}(1, \mu)-\left(\beta_{2}^{\prime} \mu^{2}+\beta_{2}\right) \mathfrak{y}^{\prime}(1, \mu)=0
\end{gathered}
$$

and transmission conditions

$$
\begin{aligned}
& \mathscr{L}_{3}(\mathfrak{y}):=\gamma_{1} \mathfrak{y}\left(0^{-}, \mu\right)-\delta_{1} \mathfrak{y}\left(0^{+}, \mu\right)=0, \\
& \mathscr{L}_{4}(\mathfrak{y}):=\gamma_{2} \mathfrak{y}^{\prime}\left(0^{-}, \mu\right)-\delta_{2} \mathfrak{y}^{\prime}\left(0^{+}, \mu\right)=0,
\end{aligned}
$$

where $\mu$ is a complex spectral parameter; $q(x)$ is a given realvalued function, which is continuous in $[-1,0)$ and $(0,1]$ and has a finite limit $q\left(0^{ \pm}\right)=\lim _{x \rightarrow 0^{ \pm}} q(x) ; \gamma_{i}, \delta_{i}, \alpha_{i}, \beta_{i}, \alpha_{i}^{\prime}$, and $\beta_{i}^{\prime}$ $(i=1,2)$ are real numbers; $\gamma_{i} \neq 0, \delta_{i} \neq 0(i=1,2) ; \gamma_{1} \gamma_{2}=\delta_{1} \delta_{2}$; and

$$
\operatorname{det}\left(\begin{array}{ll}
\alpha_{1}^{\prime} & \alpha_{1} \\
\alpha_{2}^{\prime} & \alpha_{2}
\end{array}\right)>0, \quad \operatorname{det}\left(\begin{array}{ll}
\beta_{1}^{\prime} & \beta_{1} \\
\beta_{2}^{\prime} & \beta_{2}
\end{array}\right)>0
$$

The eigenvalue problem (3)-(6) will be denoted by $\Pi(q, \alpha$, $\left.\beta, \alpha^{\prime}, \beta^{\prime}, \gamma, \delta\right)$ when $\left(\alpha_{1}^{\prime}, \alpha_{2}^{\prime}\right) \neq(0,0) \neq\left(\beta_{1}^{\prime}, \beta_{2}^{\prime}\right)$. It is a SturmLiouville problem which contains an eigenparameter $\mu$ in two boundary conditions, in addition to an internal point of discontinuity.

This approach is a fully new technique that uses the recently obtained estimates for the truncation and amplitude errors associated with (1) (cf. [23]). Both types of errors normally appear in numerical techniques that use interpolation procedures. In the following we summarize these estimates. The truncation error associated with (1) is defined to be

$$
\mathscr{R}_{N}(f)(t):=f(t)-f_{N}(t), \quad N \in \mathbb{Z}^{+}, t \in \mathbb{R}
$$

where $f_{N}(t)$ is the truncated series

$$
\begin{aligned}
& f_{N}(t) \\
& \quad=\sum_{|n| \leq N}\left[f\left(\frac{n \pi}{\sigma}\right) S_{n}^{2}(t)+f^{\prime}\left(\frac{n \pi}{\sigma}\right) \frac{\sin (\sigma t-n \pi)}{\sigma} S_{n}(t)\right] .
\end{aligned}
$$

It is proved in [23] that if $f(t) \in B_{\sigma}^{2}$ and $f(t)$ is sufficiently smooth in the sense that there exists $k \in \mathbb{Z}^{+}$such that $t^{k} f(t) \in$ $L^{2}(\mathbb{R})$, then, for $t \in \mathbb{R},|t|<N \pi / \sigma$, we have

$$
\begin{aligned}
\left|\mathscr{R}_{N}(f)(t)\right| \leq & \mathscr{T}_{N, k, \sigma}(t) \\
:= & \frac{\eta_{k, \sigma} \mathscr{E}_{k}|\sin \sigma t|^{2}}{\sqrt{3}(N+1)^{k}} \\
& \times\left(\frac{1}{(N \pi-\sigma t)^{3 / 2}}+\frac{1}{(N \pi+\sigma t)^{3 / 2}}\right) \\
& +\frac{\eta_{k, \sigma}\left(\sigma \mathscr{E}_{k}+k \mathscr{E}_{k-1}\right)|\sin \sigma t|^{2}}{\sigma(N+1)^{k}} \\
& \times\left(\frac{1}{\sqrt{N \pi-\sigma t}}+\frac{1}{\sqrt{N \pi+\sigma t}}\right),
\end{aligned}
$$

where the constants $\mathscr{E}_{k}$ and $\eta_{k, \sigma}$ are given by

$$
\begin{gathered}
\mathscr{E}_{k}:=\sqrt{\int_{-\infty}^{\infty}\left|t^{k} f(t)\right|^{2} d t} \\
\eta_{k, \sigma}:=\frac{\sigma^{k+1 / 2}}{\pi^{k+1} \sqrt{1-4^{-k}}} .
\end{gathered}
$$

The amplitude error occurs when approximate samples are used instead of the exact ones, which we cannot compute. It is defined to be

$$
\begin{aligned}
\mathscr{A}(\varepsilon, f)(t) & \\
=\sum_{n=-\infty}^{\infty}[ & \left\{f\left(\frac{n \pi}{\sigma}\right)-\widetilde{f}\left(\frac{n \pi}{\sigma}\right)\right\} S_{n}^{2}(t) \\
& +\left\{f^{\prime}\left(\frac{n \pi}{\sigma}\right)-\widetilde{f^{\prime}}\left(\frac{n \pi}{\sigma}\right)\right\} \\
& \left.\times \frac{\sin (\sigma t-n \pi)}{\sigma} S_{n}(t)\right], \quad t \in \mathbb{R},
\end{aligned}
$$

where $\tilde{f}(n \pi / \sigma)$ and $\widetilde{f^{\prime}}(n \pi / \sigma)$ are approximate samples of $f(n \pi / \sigma)$ and $f^{\prime}(n \pi / \sigma)$, respectively. Let us assume that the differences $\varepsilon_{n}:=f(n \pi / \sigma)-\widetilde{f}(n \pi / \sigma), \varepsilon_{n}^{\prime}:=f^{\prime}(n \pi / \sigma)-$ $\widetilde{f^{\prime}}(n \pi / \sigma), n \in \mathbb{Z}$, are bounded by a positive number $\varepsilon$; that is, $\left|\varepsilon_{n}\right|,\left|\varepsilon_{n}^{\prime}\right| \leq \varepsilon$. If $f(t) \in B_{\sigma}^{2}$ satisfies the natural decay conditions

$$
\begin{gathered}
\left|\varepsilon_{n}\right| \leq\left|f\left(\frac{n \pi}{\sigma}\right)\right|, \quad\left|\varepsilon_{n}^{\prime}\right| \leq\left|f^{\prime}\left(\frac{n \pi}{\sigma}\right)\right|, \\
|f(t)| \leq \frac{\mathscr{M}_{f}}{|t|^{\alpha+1}}, \quad t \in \mathbb{R}-\{0\},
\end{gathered}
$$


$0<\alpha \leq 1$, then, for $0<\varepsilon \leq \min \{\pi / \sigma, \sigma / \pi, 1 / \sqrt{e}\}$, we have [23]

$$
\begin{aligned}
\|\mathscr{A}(\varepsilon, f)\|_{\infty} & \\
\leq & \frac{4 e^{1 / 4}}{\sigma(\alpha+1)} \\
& \times\left\{\sqrt{3} e(1+\sigma)+\left((\pi / \sigma) A+\mathscr{M}_{f}\right) \rho(\varepsilon)\right. \\
& \left.+(\sigma+2+\log (2)) \mathscr{M}_{f}\right\} \varepsilon \log \left(\frac{1}{\varepsilon}\right),
\end{aligned}
$$

where

$$
\begin{gathered}
A:=\frac{3 \sigma}{\pi}\left(|f(0)|+\mathscr{M}_{f}\left(\frac{\sigma}{\pi}\right)^{\alpha}\right), \\
\rho(\varepsilon):=\gamma+10 \log \left(\frac{1}{\varepsilon}\right),
\end{gathered}
$$

and $\gamma:=\lim _{n \rightarrow \infty}\left[\sum_{k=1}^{n}(1 / k)-\log n\right] \cong 0.577216$ is the EulerMascheroni constant.

The classical [24] sampling theorem of WKS for $f \in B_{\sigma}^{2}$ is the series representation

$$
f(t)=\sum_{n=-\infty}^{\infty} f\left(\frac{n \pi}{\sigma}\right) S_{n}(t), \quad t \in \mathbb{R},
$$

where the convergence is absolute and uniform on $\mathbb{R}$ and it is uniform on compact sets of $\mathbb{C}$ (cf. [24-26]). Series (17), which is of Lagrange interpolation type, has been used to compute eigenvalues of second-order eigenvalue problems; see, for example, $[8-13,15,27,28]$.

The use of (17) in numerical analysis is known as the sinc method established by Stenger et al. (cf. [29-31]). In $[9,15,28]$, the authors applied (17) and the regularized sinc method to compute eigenvalues of different boundary value problems with a derivation of the error estimates as given by $[32,33]$. In [34], the authors used Hermite-type sampling series (1) to compute the eigenvalues of Dirac system with an internal point of discontinuity. In [14], Tharwat proved that $\Pi\left(q, \alpha, \beta, \alpha^{\prime}, \beta^{\prime}, \gamma, \delta\right)$ has a denumerable set of real and simple eigenvalues.

In [35], we compute the eigenvalues of the problem $\Pi\left(q, \alpha, \beta, \alpha^{\prime}, \beta^{\prime}, \gamma, \delta\right)$ numerically by using sinc-Gaussian technique. The main aim of the present work is to compute the eigenvalues of $\Pi\left(q, \alpha, \beta, \alpha^{\prime}, \beta^{\prime}, \gamma, \delta\right)$ numerically by using Hermite interpolations with an error analysis. This method is based on sampling theorem and Hermite interpolations but applied to regularized functions, hence avoiding any (multiple) integration and keeping the number of terms in the Cardinal series manageable. It has been demonstrated that the method is capable of delivering higher order estimates of the eigenvalues at a very low cost; see [34]. Also, in this work, by using computable error bounds we obtain eigenvalue enclosures in a simple way which not have been proven in [35].

Notice that due to Paley-Wiener's theorem $f \in B_{\sigma}^{2}$ if and only if there is $g(\cdot) \in L^{2}(-\sigma, \sigma)$ such that

$$
f(t)=\frac{1}{\sqrt{2 \pi}} \int_{-\sigma}^{\sigma} g(x) e^{i x t} d x .
$$

Therefore $f^{\prime}(t) \in B_{\sigma}^{2}$; that is, $f^{\prime}(t)$ also has an expansion of the form (17). However, $f^{\prime}(t)$ can be also obtained by termby-term differentiation formula of (17)

$$
f^{\prime}(t)=\sum_{n=-\infty}^{\infty} f\left(\frac{n \pi}{\sigma}\right) S_{n}^{\prime}(t)
$$

(see [24, page 52] for convergence). Thus the use of Hermite interpolations will not cost any additional computational efforts since the samples $f(n \pi / \sigma)$ will be used to compute both $f(t)$ and $f^{\prime}(t)$ according to (17) and (19), respectively.

In the next section, we derive the Hermite interpolation technique to compute the eigenvalues of $\Pi\left(q, \alpha, \beta, \alpha^{\prime}, \beta^{\prime}, \gamma, \delta\right)$ with error estimates. The last section contains three worked examples with comparisons accompanied by figures and numerics with Lagrange interpolation method.

\section{Treatment of $\Pi\left(q, \alpha, \beta, \alpha^{\prime}, \beta^{\prime}, \gamma, \delta\right)$}

In this section we derive approximate values of the eigenvalues of $\Pi\left(q, \alpha, \beta, \alpha^{\prime}, \beta^{\prime}, \gamma, \delta\right)$. Recall that $\Pi\left(q, \alpha, \beta, \alpha^{\prime}, \beta^{\prime}, \gamma, \delta\right)$ has denumerable set of real and simple eigenvalues (cf. [14]). Let

$$
\mathfrak{y}(x, \mu)= \begin{cases}\mathfrak{y}_{1}(x, \mu), & x \in[-1,0) \\ \mathfrak{y}_{2}(x, \mu), & x \in(0,1]\end{cases}
$$

denote the solution of (3) satisfying the following initial conditions:

$$
\left(\begin{array}{ll}
\mathfrak{y}_{1}(-1, \mu) & \mathfrak{y}_{2}\left(0^{+}, \mu\right) \\
\mathfrak{y}_{1}^{\prime}(-1, \mu) & \mathfrak{y}_{2}^{\prime}\left(0^{+}, \mu\right)
\end{array}\right)=\left(\begin{array}{cc}
\mu^{2} \alpha_{2}^{\prime}-\alpha_{2} & \frac{\gamma_{1}}{\delta_{1}} \mathfrak{y}_{1}\left(0^{-}, \mu\right) \\
\mu^{2} \alpha_{1}^{\prime}-\alpha_{1} & \frac{\gamma_{2}}{\delta_{2}} \mathfrak{y}_{1}^{\prime}\left(0^{-}, \mu\right)
\end{array}\right)
$$

Since $y(\cdot, \mu)$ satisfies $(4),(6)$, then the eigenvalues of problem (3)-(6) are the zeros of the characteristic determinant (cf. [14])

$$
\Gamma(\mu):=\left(\beta_{1}^{\prime} \mu^{2}+\beta_{1}\right) \mathfrak{y}_{2}(1, \mu)-\left(\beta_{2}^{\prime} \mu^{2}+\beta_{2}\right) \mathfrak{y}_{2}^{\prime}(1, \mu)
$$

According to [14], see also [36-38], function $\Gamma(\mu)$ is an entire function of $\mu$ where zeros are real and simple. We aim to approximate $\Gamma(\mu)$ and hence its zeros, that is, the eigenvalues by the use of the sampling theorem. The idea is to split $\Gamma(\mu)$ into two parts: one is known and the other is unknown, but lies in a Paley-Wiener space. Then we approximate the unknown part using (1) to get the approximate $\Gamma(\mu)$ and then compute the approximate zeros. Using the method of 
variation of parameters, solution $y(\cdot, \mu)$ satisfies the Volterra integral equations (cf. [14])

$$
\begin{aligned}
\mathfrak{y}_{1}(x, \mu) & \\
= & \left(-\alpha_{2}+\mu^{2} \alpha_{2}^{\prime}\right) \cos [\mu(x+1)] \\
& -\left(-\alpha_{1}+\mu^{2} \alpha_{1}^{\prime}\right) \frac{1}{\mu} \sin [\mu(x+1)] \\
& +\left(\mathfrak{T}_{1} \mathfrak{y}_{1}\right)(x, \mu), \\
\mathfrak{y}_{2}(x, \mu) & =\frac{\gamma_{1}}{\delta_{1}} \mathfrak{y}_{1}\left(0^{-}, \mu\right) \cos [\mu x] \\
& +\frac{\gamma_{2}}{\delta_{2}} \mathfrak{y}_{1}^{\prime}\left(0^{-}, \mu\right) \frac{\sin [\mu x]}{\mu}+\left(\mathfrak{I}_{2} \mathfrak{y}_{2}\right)(x, \mu),
\end{aligned}
$$

where $\mathfrak{I}_{1}$ and $\mathfrak{I}_{2}$ are the Volterra operators

$$
\begin{aligned}
& \left(\mathfrak{I}_{1} \mathfrak{y}_{1}\right)(x, \mu):=\int_{-1}^{x} \frac{\sin [\mu(x-t)]}{\mu} q(t) \mathfrak{y}_{1}(t, \mu) d t, \\
& \left(\mathfrak{I}_{2} \mathfrak{y}_{2}\right)(x, \mu):=\int_{0}^{x} \frac{\sin [\mu(x-t)]}{\mu} q(t) \mathfrak{y}_{2}(t, \mu) d t .
\end{aligned}
$$

Differentiating (23) we obtain

$$
\begin{aligned}
\mathfrak{y}_{1}^{\prime}(x, \mu)= & -\left(-\alpha_{2}+\mu^{2} \alpha_{2}^{\prime}\right) \mu \sin [\mu(x+1)] \\
& -\left(-\alpha_{1}+\mu^{2} \alpha_{1}^{\prime}\right) \cos [\mu(x+1)] \\
& +\left(\widetilde{\mathfrak{T}}_{1} \mathfrak{y}_{1}\right)(x, \mu), \\
\mathfrak{y}_{2}^{\prime}(x, \mu)= & -\frac{\gamma_{1}}{\delta_{1}} \mu \mathfrak{y}_{1}\left(0^{-}, \mu\right) \sin [\mu x] \\
& +\frac{\gamma_{2}}{\delta_{2}} \mathfrak{y}_{1}^{\prime}\left(0^{-}, \mu\right) \cos [\mu x] \\
& +\left(\widetilde{\mathfrak{T}}_{2} \mathfrak{y}_{2}\right)(x, \mu),
\end{aligned}
$$

where $\widetilde{\mathfrak{I}}_{1}$ and $\widetilde{\mathfrak{I}}_{2}$ are the Volterra-type integral operators

$$
\begin{aligned}
& \left(\widetilde{\mathfrak{T}}_{1} \mathfrak{y}_{1}\right)(x, \mu):=\int_{-1}^{x} \cos [\mu(x-t)] q(t) \mathfrak{y}_{1}(t, \mu) d t, \\
& \left(\widetilde{\mathfrak{T}}_{2} \mathfrak{y}_{2}\right)(x, \mu):=\int_{0}^{x} \cos [\mu(x-t)] q(t) \mathfrak{y}_{2}(t, \mu) d t .
\end{aligned}
$$

Define $\vartheta_{i}(\cdot, \mu)$ and $\widetilde{\vartheta}_{i}(\cdot, \mu), i=1,2$, to be

$$
\vartheta_{i}(x, \mu):=\mathfrak{\mathfrak { I }}_{i} \mathfrak{y}_{i}(x, \mu), \quad \widetilde{\vartheta}_{i}(x, \mu):=\widetilde{\mathfrak{T}}_{i} \mathfrak{y}_{i}(x, \mu)
$$

In the following, we will make use of the known estimates:

$$
|\cos z| \leq e^{|\Im z|}, \quad\left|\frac{\sin z}{z}\right| \leq \frac{c_{0}}{1+|z|} e^{|\Im z|}
$$

where $c_{0}$ is some constant (we may take $c_{0} \simeq 1.72$ ). For convenience, we define the constants

$$
\begin{gathered}
q_{1}:=\int_{-1}^{0} q(t) d t, \quad q_{2}:=\int_{0}^{1} q(t) d t, \\
c_{1}:=\max \left(\left|\alpha_{1}\right|,\left|\alpha_{2}\right|,\left|\alpha_{1}^{\prime}\right|,\left|\alpha_{2}^{\prime}\right|\right), \quad c_{2}:=\exp \left(c_{0} q_{1}\right), \\
c_{3}:=1+c_{0} c_{2} q_{1}, \\
c_{4}:=\left(1+c_{0}\right)\left[\frac{\left|\gamma_{1}\right|}{\left|\delta_{1}\right|} c_{3}+\frac{\left|\gamma_{2}\right|}{\left|\delta_{2}\right|} c_{0}\left(1+c_{3} q_{1}\right)\right], \\
c_{5}:=\exp \left(c_{0} q_{2}\right), \quad c_{6}:=1+c_{0} q_{2} c_{5} .
\end{gathered}
$$

As in [15] we split $\Gamma(\mu)$ into two parts via

$$
\Gamma(\mu):=\mathscr{G}(\mu)+\mathcal{S}(\mu),
$$

where $\mathscr{G}(\mu)$ is the known part

$$
\begin{aligned}
\mathscr{G}(\mu):= & \left(\beta_{1}^{\prime} \mu^{2}+\beta_{1}\right) \\
\times & {\left[\left(\mu^{2} \alpha_{2}^{\prime}-\alpha_{2}\right)\left(\frac{\gamma_{1}}{\delta_{1}} \cos ^{2} \mu-\frac{\gamma_{2}}{\delta_{2}} \sin ^{2} \mu\right)\right.} \\
& \left.\quad-\left(\mu^{2} \alpha_{1}^{\prime}-\alpha_{1}\right)\left(\frac{\gamma_{1}}{\delta_{1}}+\frac{\gamma_{2}}{\delta_{2}}\right) \cos \mu \frac{\sin \mu}{\mu}\right] \\
+ & \left(\beta_{2}^{\prime} \mu^{2}+\beta_{2}\right) \\
\times & {\left[\left(\mu^{2} \alpha_{2}^{\prime}-\alpha_{2}\right)\left(\frac{\gamma_{1}}{\delta_{1}}+\frac{\gamma_{2}}{\delta_{2}}\right) \mu \cos \mu \sin \mu\right.} \\
& \left.\quad+\left(\mu^{2} \alpha_{1}^{\prime}-\alpha_{1}\right)\left(\frac{\gamma_{2}}{\delta_{2}} \cos ^{2} \mu-\frac{\gamma_{1}}{\delta_{1}} \sin ^{2} \mu\right)\right]
\end{aligned}
$$

and $\mathcal{S}(\mu)$ is the unknown one

$$
\begin{aligned}
\mathcal{S}(\mu):= & \frac{\gamma_{1}}{\delta_{1}} \\
& \times\left[\left(\beta_{1}^{\prime} \mu^{2}+\beta_{1}\right) \cos \mu+\left(\beta_{2}^{\prime} \mu^{2}+\beta_{2}\right) \mu \sin \mu\right] \\
& \times \vartheta_{1}\left(0^{-}, \mu\right) \\
& +\left(\beta_{1}^{\prime} \mu^{2}+\beta_{1}\right) \vartheta_{2}(1, \mu) \\
& +\frac{\gamma_{2}}{\delta_{2}}\left[\left(\beta_{1}^{\prime} \mu^{2}+\beta_{1}\right) \frac{\sin \mu}{\mu}-\left(\beta_{2}^{\prime} \mu^{2}+\beta_{2}\right) \cos \mu\right] \\
& \times \widetilde{\vartheta}_{1}\left(0^{-}, \mu\right) \\
& -\left(\beta_{2}^{\prime} \mu^{2}+\beta_{2}\right) \widetilde{\vartheta}_{2}(1, \mu) .
\end{aligned}
$$

Then function $\delta(\mu)$ is entire in $\mu$ for each $x \in[0,1]$ for which (cf. [15])

$$
|\mathcal{S}(\mu)| \leq \mathscr{M}\left(1+|\mu|^{2}\right)^{2} e^{2|\Im \mu|}, \quad \mu \in \mathbb{C},
$$


where

$$
\begin{gathered}
\mathscr{M}:=c_{1} c\left(1+c_{0}\right)^{2} q_{1}\left[c_{0} c_{2} \frac{\left|\gamma_{1}\right|}{\left|\delta_{1}\right|}+c_{3} \frac{\left|\gamma_{2}\right|}{\left|\delta_{2}\right|}\right]+c_{1} c_{4} c q_{2}\left(c_{6}+c_{0} c_{5}\right), \\
c:=\max \left\{\left|\beta_{1}\right|,\left|\beta_{2}\right|,\left|\beta_{1}^{\prime}\right|,\left|\beta_{2}^{\prime}\right|\right\} .
\end{gathered}
$$

The analyticity of $\delta(\mu)$ as well as estimate (33) is not adequate to prove that $\delta(\mu)$ lies in a Paley-Wiener space. To solve this problem, we will multiply $\delta(\mu)$ by a regularization factor. Let $\theta \in(0,1)$ and $m \in \mathbb{Z}^{+}, m>5$, be fixed. Let $\mathscr{F}_{\theta, m}(\mu)$ be the function

$$
\mathscr{F}_{\theta, m}(\mu):=\left(\frac{\sin \theta \mu}{\theta \mu}\right)^{m} \mathcal{S}(\mu), \quad \mu \in \mathbb{C} .
$$

The regularizing factor has been introduced in [9], in the context of the regularized sampling method, which was used in [9-13] to compute the eigenvalues of several classes of Sturm-Liouville problems. More specifications on $m, \theta$ will be given latter on. Then $\mathscr{F}_{\theta, m}(\mu)$, see [15], is an entire function of $\mu$ which satisfies the estimate

$$
\left|\mathscr{F}_{\theta, m}(\mu)\right| \leq \frac{\mathscr{M} c_{0}^{m}\left(1+|\mu|^{2}\right)^{2}}{(1+\theta|\mu|)^{m}} e^{|\widetilde{\Im} \mu|(2+m \theta)}, \quad \mu \in \mathbb{C} .
$$

Moreover, $\mu^{m-5} \mathscr{F}_{\theta, m}(\mu) \in L^{2}(\mathbb{R})$ and

$$
\mathscr{E}_{m-5}\left(\mathscr{F}_{\theta, m}\right):=\sqrt{\int_{-\infty}^{\infty}\left|\mu^{m-5} \mathscr{F}_{\theta, m}(\mu)\right|^{2} d \mu} \leq \sqrt{2 v_{0}} \mu c_{0}^{m},
$$

where

$$
\begin{aligned}
v_{0}:=\frac{1}{\theta^{2 m-1}} & \\
& \times\left(\frac{1}{2 m-1}+\frac{4 \theta^{2}}{4 m^{3}-12 m^{2}+11 m-3}\right. \\
& +\left(144 \theta^{4}\left(280 \theta^{4} \Gamma[2 m-9]+20 \theta^{2} \Gamma[2 m-7]\right)\right. \\
& \quad+\Gamma[2 m-5]) \\
& \left.\quad \times(\Gamma[2 m])^{-1}\right) .
\end{aligned}
$$

What we have just proved is that $\mathscr{F}_{\theta, m}(\mu)$ belongs to the Paley-Wiener space $B_{\sigma}^{2}$ with $\sigma=2+m \theta$. Since $\mathscr{F}_{\theta, m}(\mu) \epsilon$ $B_{\sigma}^{2} \subset B_{2 \sigma}^{2}$, then we can reconstruct the functions $\mathscr{F}_{\theta, m}(\mu)$ via the following sampling formula:

$$
\begin{aligned}
\mathscr{F}_{\theta, m}(\mu) & \\
=\sum_{n=-\infty}^{\infty}[ & \mathscr{F}_{\theta, m}\left(\frac{n \pi}{\sigma}\right) S_{n}^{2}(\mu) \\
& \left.+\mathscr{F}_{\theta, m}^{\prime}\left(\frac{n \pi}{\sigma}\right) \frac{\sin (\sigma \mu-n \pi)}{\sigma} S_{n}(\mu)\right] .
\end{aligned}
$$

Let $N \in \mathbb{Z}^{+}, N>m$, and approximate $\mathscr{F}_{\theta, m}(\mu)$ by its truncated series $\mathscr{F}_{\theta, m, N}(\mu)$, where

$$
\begin{aligned}
\mathscr{F}_{\theta, m, N}(\mu) & =\sum_{n=-N}^{N}\left[\mathscr{F}_{\theta, m}\left(\frac{n \pi}{\sigma}\right) S_{n}^{2}(\mu)\right. \\
& \left.\quad+\mathscr{F}_{\theta, m}^{\prime}\left(\frac{n \pi}{\sigma}\right) \frac{\sin (\sigma \mu-n \pi)}{\sigma} S_{n}(\mu)\right] .
\end{aligned}
$$

Since all eigenvalues are real, then from now on we restrict ourselves to $\mu \in \mathbb{R}$. Since $\mu^{m-5} \mathscr{F}_{\theta, m}(\mu) \in L^{2}(\mathbb{R})$, the truncation error (cf. (10)) is given for $|\mu|<N \pi / \sigma$ by

$$
\left|\mathscr{F}_{\theta, m}(\mu)-\mathscr{F}_{\theta, m, N}(\mu)\right| \leq T_{N, m-5, \sigma}(\mu),
$$

where

$$
\begin{aligned}
\mathscr{T}_{N, m-5, \sigma} & (\mu) \\
:= & \frac{\eta_{m-5, \sigma} \mathscr{E}_{m-5}|\sin \sigma \mu|^{2}}{\sqrt{3}(N+1)^{m-5}} \\
& \times\left(\frac{1}{(N \pi-\sigma \mu)^{3 / 2}}+\frac{1}{(N \pi+\sigma \mu)^{3 / 2}}\right) \\
& +\frac{\eta_{m-5, \sigma}\left(\sigma \mathscr{E}_{m-5}+(m-5) \mathscr{E}_{m-6}\right)|\sin \sigma \mu|^{2}}{\sigma(N+1)^{m-5}} \\
& \times\left(\frac{1}{\sqrt{N \pi-\sigma \mu}}+\frac{1}{\sqrt{N \pi+\sigma \mu}}\right) .
\end{aligned}
$$

The samples $\left\{\mathscr{F}_{\theta, m}(n \pi / \sigma)\right\}_{n=-N}^{N}$ and $\left\{\mathscr{F}_{\theta, m}^{\prime}(n \pi / \sigma)\right\}_{n=-N}^{N}$, in general, are not known explicitly. So we approximate them by solving numerically $8 N+4$ initial value problems at the nodes $\{n \pi / \sigma\}_{n=-N}^{N}$.

Let $\left\{\widetilde{\mathscr{F}}_{\theta, m}(n \pi / \sigma)\right\}_{n=-N}^{N}$ and $\left\{\widetilde{\mathscr{F}}_{\theta, m}^{\prime}(n \pi / \sigma)\right\}_{n=-N}^{N}$ be the approximations of the samples of $\left\{\mathscr{F}_{\theta, m}(n \pi / \sigma)\right\}_{n=-N}^{N}$ and $\left\{\mathscr{F}_{\theta, m}^{\prime}(n \pi / \sigma)\right\}_{n=-N}^{N}$, respectively. Now we define $\widetilde{\mathscr{F}}_{\theta, m, N}(\mu)$, which approximates $\mathscr{F}_{\theta, m, N}(\mu)$ :

$$
\begin{aligned}
\widetilde{\mathscr{F}}_{\theta, m, N}(\mu) & \\
:=\sum_{n=-N}^{N}[ & \widetilde{\mathscr{F}}_{\theta, m}\left(\frac{n \pi}{\sigma}\right) S_{n}^{2}(\mu) \\
& +\widetilde{\mathscr{F}}_{\theta, m}^{\prime}\left(\frac{n \pi}{\sigma}\right) \\
& \left.\times \frac{\sin (\sigma \mu-n \pi)}{\sigma} S_{n}(\mu)\right], \quad N>m .
\end{aligned}
$$

Using standard methods for solving initial problems, we may assume that for $|n|<N$

$$
\begin{aligned}
& \left|\mathscr{F}_{\theta, m}\left(\frac{n \pi}{\sigma}\right)-\widetilde{\mathscr{F}}_{\theta, m}\left(\frac{n \pi}{\sigma}\right)\right|<\varepsilon, \\
& \left|\mathscr{F}_{\theta, m}^{\prime}\left(\frac{n \pi}{\sigma}\right)-\widetilde{\mathscr{F}}_{\theta, m}^{\prime}\left(\frac{n \pi}{\sigma}\right)\right|<\varepsilon,
\end{aligned}
$$


for a sufficiently small $\varepsilon$. From $(36)$ we can see that $\mathscr{F}_{\theta, m}(\mu)$ satisfies the condition (14) when $m>5$, and therefore whenever $0<\varepsilon \leq \min \{\pi / \sigma, \sigma / \pi, 1 / \sqrt{e}\}$ we have

$$
\left|\mathscr{F}_{\theta, m, N}(\mu)-\widetilde{\mathscr{F}}_{\theta, m, N}(\mu)\right| \leq \mathscr{A}(\varepsilon), \quad \mu \in \mathbb{R},
$$

where there is a positive constant $M_{\mathscr{F}_{\theta, m}}$ for which (cf. (15))

$$
\begin{aligned}
\mathscr{A}(\varepsilon):= & \frac{2 e^{1 / 4}}{\sigma} \\
& \times\left\{\sqrt{3} e(1+\sigma)+\left(\frac{\pi}{\sigma} A+\mathscr{M}_{\mathscr{F}_{\theta, m}}\right) \rho(\varepsilon)\right. \\
& \left.+(\sigma+2+\log (2)) \mathscr{M}_{\mathscr{F}_{\theta, m}}\right\} \varepsilon \log \left(\frac{1}{\varepsilon}\right) .
\end{aligned}
$$

Here

$$
\begin{gathered}
A:=\frac{3 \sigma}{\pi}\left(\left|\mathscr{F}_{\theta, m}(0)\right|+\frac{\sigma}{\pi} \mathscr{M}_{\mathscr{F}_{\theta, m}}\right), \\
\rho(\varepsilon):=\gamma+10 \log \left(\frac{1}{\varepsilon}\right) .
\end{gathered}
$$

In the following we use the technique of [27], see also [15], to determine enclosure intervals for the eigenvalues. Let $\mu^{*}$ be an eigenvalue; that is,

$$
\Gamma\left(\mu^{*}\right)=\mathscr{G}\left(\mu^{*}\right)+\left(\frac{\sin \theta \mu^{*}}{\theta \mu^{*}}\right)^{-m} \mathscr{F}_{\theta, m}\left(\mu^{*}\right)=0 .
$$

Then it follows that

$$
\begin{aligned}
\mathscr{G}\left(\mu^{*}\right)+ & \left(\frac{\sin \theta \mu^{*}}{\theta \mu^{*}}\right)^{-m} \widetilde{\mathscr{F}}_{\theta, m, N}\left(\mu^{*}\right) \\
= & \left(\frac{\sin \theta \mu^{*}}{\theta \mu^{*}}\right)^{-m} \widetilde{\mathscr{F}}_{\theta, m, N}\left(\mu^{*}\right) \\
& -\left(\frac{\sin \theta \mu^{*}}{\theta \mu^{*}}\right)^{-m} \mathscr{F}_{\theta, m}\left(\mu^{*}\right) \\
= & {\left[\left(\frac{\sin \theta \mu^{*}}{\theta \mu^{*}}\right)^{-m} \widetilde{\mathscr{F}}_{\theta, m, N}\left(\mu^{*}\right)\right.} \\
& \left.-\left(\frac{\sin \theta \mu^{*}}{\theta \mu^{*}}\right)^{-m} \mathscr{F}_{\theta, m, N}\left(\mu^{*}\right)\right] \\
+ & {\left[\left(\frac{\sin \theta \mu^{*}}{\theta \mu^{*}}\right)^{-m} \mathscr{F}_{\theta, m, N}\left(\mu^{*}\right)\right.} \\
& \left.-\left(\frac{\sin \theta \mu^{*}}{\theta \mu^{*}}\right)^{-m} \mathscr{F}_{\theta, m}\left(\mu^{*}\right)\right]
\end{aligned}
$$

and so

$$
\begin{aligned}
& \left|\mathscr{G}\left(\mu^{*}\right)+\left(\frac{\sin \theta \mu^{*}}{\theta \mu^{*}}\right)^{-m} \widetilde{\mathscr{F}}_{\theta, m, N}\left(\mu^{*}\right)\right| \\
& \quad \leq\left|\frac{\sin \theta \mu^{*}}{\theta \mu^{*}}\right|^{-m}\left(T_{N, m-5, \sigma}\left(\mu^{*}\right)+\mathscr{A}(\varepsilon)\right) .
\end{aligned}
$$

Since

$$
\mathscr{G}\left(\mu^{*}\right)+\left(\frac{\sin \theta \mu^{*}}{\theta \mu^{*}}\right)^{-m} \widetilde{\mathscr{F}}_{\theta, m, N}\left(\mu^{*}\right)
$$

is given and

$$
\left|\frac{\sin \theta \mu^{*}}{\theta \mu^{*}}\right|^{-m}\left(T_{N, m-5, \sigma}\left(\mu^{*}\right)+\mathscr{A}(\varepsilon)\right)
$$

has computable upper bound, we can define an enclosure for $\mu^{*}$, by solving the following system of inequalities:

$$
\begin{gathered}
-\left|\frac{\sin \theta \mu^{*}}{\theta \mu^{*}}\right|^{-m}\left(\mathscr{T}_{N, m-5, \sigma}\left(\mu^{*}\right)+\mathscr{A}(\varepsilon)\right) \\
\leq \mathscr{G}\left(\mu^{*}\right)+\left(\frac{\sin \theta \mu^{*}}{\theta \mu^{*}}\right)^{-m} \widetilde{\mathscr{F}}_{\theta, m, N}\left(\mu^{*}\right) \\
\leq\left|\frac{\sin \theta \mu^{*}}{\theta \mu^{*}}\right|^{-m}\left(\mathscr{T}_{N, m-5, \sigma}\left(\mu^{*}\right)+\mathscr{A}(\varepsilon)\right) .
\end{gathered}
$$

Its solution is an interval containing $\mu^{*}$ over which the graph $\mathscr{G}\left(\mu^{*}\right)+\left(\sin \theta \mu^{*} / \theta \mu^{*}\right)^{-m} \widetilde{\mathscr{F}}_{\theta, m, N}\left(\mu^{*}\right)$ is squeezed between the graphs

$$
\begin{gathered}
-\left|\frac{\sin \theta \mu^{*}}{\theta \mu^{*}}\right|^{-m}\left(\mathscr{T}_{N, m-5, \sigma}\left(\mu^{*}\right)+\mathscr{A}(\varepsilon)\right), \\
\left|\frac{\sin \theta \mu^{*}}{\theta \mu^{*}}\right|^{-m}\left(\mathscr{T}_{N, m-5, \sigma}\left(\mu^{*}\right)+\mathscr{A}(\varepsilon)\right) .
\end{gathered}
$$

Using the fact that

$$
\widetilde{\mathscr{F}}_{\theta, m, N}(\mu) \longrightarrow \mathscr{F}_{\theta, m}(\mu),
$$

uniformly over any compact set, and since $\mu^{*}$ is a simple root, we obtain for large $N$ and sufficiently small $\varepsilon$

$$
\frac{\partial}{\partial \mu}\left(\mathscr{G}(\mu)+\left(\frac{\sin \theta \mu}{\theta \mu}\right)^{-m} \widetilde{\mathscr{F}}_{\theta, m, N}(\mu)\right) \neq 0,
$$

in a neighborhood of $\mu^{*}$. Hence the graph of

$$
\mathscr{G}(\mu)+\left(\frac{\sin \theta \mu}{\theta \mu}\right)^{-m} \widetilde{\mathscr{F}}_{\theta, m, N}(\mu)
$$

intersects the graphs

$$
\begin{gathered}
-\left|\frac{\sin \theta \mu}{\theta \mu}\right|^{-m}\left(\mathscr{T}_{N, m-5, \sigma}(\mu)+\mathscr{A}(\varepsilon)\right), \\
\left|\frac{\sin \theta \mu}{\theta \mu}\right|^{-m}\left(\mathscr{T}_{N, m-5, \sigma}(\mu)+\mathscr{A}(\varepsilon)\right),
\end{gathered}
$$

at two points with abscissae $a_{-}\left(\mu^{*}, N, \varepsilon\right) \leq a_{+}\left(\mu^{*}, N, \varepsilon\right)$, and the solution of the system of inequalities (53) is the interval

$$
I_{\varepsilon, N}:=\left[a_{-}\left(\mu^{*}, N, \varepsilon\right), a_{+}\left(\mu^{*}, N, \varepsilon\right)\right]
$$

and in particular $\mu^{*} \in I_{\varepsilon, N}$. Summarizing the above discussion, we arrive at the following lemma which is similar to that of [27] for Sturm-Liouville problems. 
Lemma 1. For any eigenvalue $\mu^{*}$, one can find $N_{0} \in \mathbb{Z}^{+}$and sufficiently small $\varepsilon$ such that $\mu^{*} \in I_{\varepsilon, N}$ for $N>N_{0}$. Moreover

$$
\begin{array}{r}
{\left[a_{-}\left(\mu^{*}, N, \varepsilon\right), a_{+}\left(\mu^{*}, N, \varepsilon\right)\right] \longrightarrow\left\{\mu^{*}\right\}} \\
\text { as } N \longrightarrow \infty, \varepsilon \longrightarrow 0 .
\end{array}
$$

Proof. Since all eigenvalues of $\Pi\left(q, \alpha, \beta, \alpha^{\prime}, \beta^{\prime}, \gamma, \delta\right)$ are simple, then for large $N$ and sufficiently small $\varepsilon$ we have $(\partial /$ $\partial \mu)\left(\mathscr{G}(\mu)+(\sin \theta \mu / \theta \mu)^{-m} \widetilde{\mathscr{F}}_{\theta, m, N}(\mu)\right)>0$, in a neighborhood of $\mu^{*}$. Choose $N_{0}$ such that

$$
\begin{aligned}
\mathscr{G}(\mu) & +\left(\frac{\sin \theta \mu}{\theta \mu}\right)^{-m} \widetilde{\mathscr{F}}_{\theta, m, N_{0}}(\mu) \\
& = \pm\left|\frac{\sin \theta \mu}{\theta \mu}\right|^{-m}\left(\mathscr{T}_{N_{0}, m-5, \sigma}(\mu)+\mathscr{A}(\varepsilon)\right)
\end{aligned}
$$

has two distinct solutions which we denote by $a_{-}\left(\mu^{*}, N_{0}, \varepsilon\right) \leq$ $a_{+}\left(\mu^{*}, N_{0}, \varepsilon\right)$. The decay of $\mathscr{T}_{N, m-5, \sigma}(\mu) \rightarrow 0$ as $N \rightarrow \infty$ and $\mathscr{A}(\varepsilon) \rightarrow 0$ as $\varepsilon \rightarrow 0$ will ensure the existence of the solutions $a_{-}\left(\mu^{*}, N, \varepsilon\right)$ and $a_{+}\left(\mu^{*}, N, \varepsilon\right)$ as $N \rightarrow \infty$ and $\varepsilon \rightarrow$ 0 . For the second point we recall that $\widetilde{\mathscr{F}}_{\theta, m, N}(\mu) \rightarrow \mathscr{F}_{\theta, m}(\mu)$ as $N \rightarrow \infty$ and $\varepsilon \rightarrow 0$. Hence by taking the limit we obtain

$$
\begin{aligned}
& \mathscr{G}\left(a_{+}\left(\mu^{*}, \infty, 0\right)\right)+\left(\frac{\sin \theta \mu^{*}}{\theta \mu^{*}}\right)^{-m} \mathscr{F}_{\theta, m}\left(a_{+}\left(\mu^{*}, \infty, 0\right)\right)=0, \\
& \mathscr{G}\left(a_{-}\left(\mu^{*}, \infty, 0\right)\right)+\left(\frac{\sin \theta \mu^{*}}{\theta \mu^{*}}\right)^{-m} \mathscr{F}_{\theta, m}\left(a_{-}\left(\mu^{*}, \infty, 0\right)\right)=0 .
\end{aligned}
$$

That is $\Gamma\left(a_{+}\right)=\Gamma\left(a_{-}\right)=0$. This leads us to conclude that $a_{+}=a_{-}=\mu^{*}$ since $\mu^{*}$ is a simple root.

Let $\widetilde{\Gamma}_{N}(\mu):=\mathscr{G}(\mu)+(\sin \theta \mu / \theta \mu)^{-m} \widetilde{\mathscr{F}}_{\theta, m, N}(\mu)$. Then (41) and (45) imply

$$
\begin{array}{r}
\left|\Gamma(\mu)-\widetilde{\Gamma}_{N}(\mu)\right| \leq\left|\frac{\sin \theta \mu}{\theta \mu}\right|^{-m}\left(\mathscr{T}_{N, m-5, \sigma}(\mu)+\mathscr{A}(\varepsilon)\right), \\
|\mu|<\frac{N \pi}{\sigma},
\end{array}
$$

and $\theta$ is chosen sufficiently small for which $|\theta \mu|<\pi$. Therefore $\theta, m$ must be chosen so that for $|\mu|<N \pi / \sigma$

$$
m>5, \quad \theta \in(0,1), \quad|\theta \mu|<\pi .
$$

Let $\mu^{*}$ be an eigenvalue and let $\mu_{N}$ be its approximation. Thus $\Gamma\left(\mu^{*}\right)=0$ and $\widetilde{\Gamma}_{N}\left(\mu_{N}\right)=0$. From (63) we have $\left|\widetilde{\Gamma}_{N}\left(\mu^{*}\right)\right| \leq$ $\left|\sin \theta \mu^{*} / \theta \mu^{*}\right|^{-m}\left(\mathscr{T}_{N, m-5, \sigma}\left(\mu^{*}\right)+\mathscr{A}(\varepsilon)\right)$. Now we estimate the error $\left|\mu^{*}-\mu_{N}\right|$ for an eigenvalue $\mu^{*}$.

Theorem 2. Let $\mu^{*}$ be an eigenvalue of $\Pi\left(q, \alpha, \beta, \alpha^{\prime}, \beta^{\prime}, \gamma, \delta\right)$. For sufficiently large $N$ one has the following estimate:

$$
\left|\mu^{*}-\mu_{N}\right|<\left|\frac{\sin \theta \mu_{N}}{\theta \mu_{N}}\right|^{-m} \frac{\mathscr{T}_{N, m-5, \sigma}\left(\mu_{N}\right)+\mathscr{A}(\varepsilon)}{\inf _{\zeta \in I_{\varepsilon, N}}\left|\Gamma^{\prime}(\zeta)\right|} .
$$

Proof. Since $\Gamma\left(\mu_{N}\right)-\widetilde{\Gamma}_{N}\left(\mu_{N}\right)=\Gamma\left(\mu_{N}\right)-\Gamma\left(\mu^{*}\right)$, then from (63) and after replacing $\mu$ by $\mu_{N}$ we obtain

$$
\left|\Gamma\left(\mu_{N}\right)-\Gamma\left(\mu^{*}\right)\right| \leq\left|\frac{\sin \theta \mu_{N}}{\theta \mu_{N}}\right|^{-m}\left(\mathscr{T}_{N, m-5, \sigma}\left(\mu_{N}\right)+\mathscr{A}(\varepsilon)\right) .
$$

Using the mean value theorem yields that for some $\zeta \in J_{\varepsilon, N}:=$ $\left[\min \left(\mu^{*}, \mu_{N}\right), \max \left(\mu^{*}, \mu_{N}\right)\right]$

$$
\begin{array}{r}
\left|\left(\mu^{*}-\mu_{N}\right) \Gamma^{\prime}(\zeta)\right| \leq\left|\frac{\sin \theta \mu_{N}}{\theta \mu_{N}}\right|^{-m}\left(\mathscr{T}_{N, m-5, \sigma}\left(\mu_{N}\right)+\mathscr{A}(\varepsilon)\right), \\
\zeta \in J_{\varepsilon, N} \subset I_{\varepsilon, N} .
\end{array}
$$

Since thlarge $\operatorname{Ninf}_{\zeta \in I_{\varepsilon, N}}\left|\Gamma^{\prime}(\zeta)\right|>0$ and we get (65).

\section{Numerical Examples}

This section includes three detailed worked examples illustrating the above technique. By $E_{S}$ and $E_{H}$ we mean the absolute errors associated with the results of the classical sinc method $[9,15]$ and our new method (Hermite interpolations), respectively. The first two examples are computed in [15] with the classical sinc method. We indicate in these two examples the effect of the amplitude error in the method by determining enclosure intervals for different values of $\varepsilon$. We also indicate the effect of the parameters $m$ and $\theta$ by several choices. Also, ine eigenvalues are simple, then for sufficiently the following two examples, we observe that the exact solutions $\mu_{k}$ and the zeros of $\Gamma(\mu)$ are all inside the interval $\left[a_{-}, a_{+}\right]$. In the third example, we compare our new method with the classical sinc method [9]. We would like to mention that MATHEMATICA has been used to obtain the exact values for the two examples where eigenvalues cannot be computed concretely. MATHEMATICA is also used in rounding the exact eigenvalues, which are square roots. Both numerical results and the associated figures prove the credibility of the method.

Recall that $a_{ \pm}(\mu)$ are defined by

$$
\begin{array}{r}
a_{ \pm}(\mu)=\widetilde{\Gamma}_{N}(\mu) \pm\left|\frac{\sin \theta \mu}{\theta \mu}\right|^{-m}\left(\mathscr{T}_{N, m-5, \sigma}(\mu)+\mathscr{A}(\varepsilon)\right), \\
|\mu|<\frac{N \pi}{\sigma} .
\end{array}
$$

Recall also that the enclosure interval $I_{\varepsilon, N}:=\left[a_{-}, a_{+}\right]$is determined by solving

$$
a_{ \pm}(\mu)=0, \quad|\mu|<\frac{N \pi}{\sigma} .
$$

Example 1. Consider the boundary value problem [15]

$$
\begin{gathered}
-\mathfrak{y}^{\prime \prime}(x, \mu)+q(x) \mathfrak{y}(x, \mu)=\mu^{2} \mathfrak{y}(x, \mu), \\
x \in[-1,0) \cup(0,1], \\
\mu^{2} \mathfrak{y}(-1, \mu)+\mathfrak{y}^{\prime}(-1, \mu)=0, \quad \mu^{2} \mathfrak{y}(1, \mu)-\mathfrak{y}^{\prime}(1, \mu)=0, \\
\mathfrak{y}\left(0^{-}, \mu\right)-\mathfrak{y}\left(0^{+}, \mu\right)=0, \quad \mathfrak{y}^{\prime}\left(0^{-}, \mu\right)-\mathfrak{y}^{\prime}\left(0^{+}, \mu\right)=0 .
\end{gathered}
$$


TABle 1: Comparing the exact, sinc, and Hermite solutions at $N=20, m=8$, and $\theta=1 / 6$.

\begin{tabular}{lccc}
\hline$\mu_{k}$ & Sinc $\mu_{k, N}[15]$ & Exact $\mu_{k}$ & Hermite $\mu_{k, N}$ \\
\hline$\mu_{1}$ & 0.5796031003909555 & 0.579603114810978 & 0.5796031148176034 \\
$\mu_{2}$ & 1.7849838911323737 & 1.7849838948888357 & 1.7849838948886403 \\
$\mu_{3}$ & 3.2386473557238586 & 3.238647349751419 & 3.238647349751933 \\
$\mu_{4}$ & 4.7705623346546995 & 4.770562335590527 & 4.770562335590518 \\
$\mu_{5}$ & 6.32570436013402 & 6.3257043646722515 & 6.325704364672412 \\
\hline
\end{tabular}

TABLE 2: Comparing the exact, sinc, and Hermite solutions at $N=20, m=12$, and $\theta=1 / 4$.

\begin{tabular}{lccr}
\hline$\mu_{k}$ & Sinc $\mu_{k, N}[15]$ & Exact $\mu_{k}$ & Hermite $\mu_{k, N}$ \\
\hline$\mu_{1}$ & 0.5796031148103449 & 0.57960311481097786078 & 0.57960311481097786304 \\
$\mu_{2}$ & 1.7849838948900418 & 1.78498389488883561160 & 1.78498389488883560884 \\
$\mu_{3}$ & 3.2386473497526262 & 3.23864734975141899542 & 3.238647349751418996887 \\
$\mu_{4}$ & 4.77056233558413 & 4.77056233559052749760 & 4.77056233559052748750 \\
$\mu_{5}$ & 6.325704364662005 & 6.32570436467225155153 & 6.32570436467225155387 \\
\hline
\end{tabular}

Here $\beta_{1}^{\prime}=\beta_{2}=\alpha_{1}^{\prime}=\alpha_{2}=1, \beta_{1}=\beta_{2}^{\prime}=\alpha_{1}=\alpha_{2}^{\prime}=0$, $\gamma_{1}=\delta_{1}=2, \gamma_{2}=\delta_{2}=1 / 2$, and

$$
q(x)= \begin{cases}-1, & x \in[-1,0), \\ -2, & x \in(0,1] .\end{cases}
$$

The characteristic function is

$$
\begin{gathered}
\Gamma(\mu)=\frac{1}{\sqrt{1+\mu^{2}} \sqrt{2+\mu^{2}}} \\
\times\left[\operatorname { s i n } \sqrt { 1 + \mu ^ { 2 } } \left(-\sqrt{2+\mu^{2}}\left(\mu^{4}-\mu^{2}-1\right)\right.\right. \\
\quad \times \cos \sqrt{2+\mu^{2}} \\
-\sqrt{1+\mu^{2}} \cos \sqrt{1+\mu^{2}} \\
\quad \times\left(-2 \mu^{2} \sqrt{2+\mu^{2}} \cos \sqrt{2+\mu^{2}} \sqrt{2+\mu^{2}}\right) \\
\left.\left.\quad+\left(\mu^{4}-\mu^{2}-2\right) \sin \sqrt{2+\mu^{2}}\right)\right] .
\end{gathered}
$$

The function $\mathscr{K}(\mu)$ will be

$$
\mathscr{K}(\mu)=-\mu\left(1+\mu^{2}\right) \sin 2 \mu .
$$

The application of Hermite interpolations method and sinc method [15] to this problem and the effect of $\theta$ and $m$ at $N=20$ are indicated in Tables 1 and 2. In Tables 3 and 4, we display the maximum absolute error of $\mu_{k}-\mu_{k, N}$, using Hermite interpolations method and sinc method [15] with various choices of $\theta$ and $m$ at $N=20$. From these tables, it is shown that the proposed methods are significantly more accurate than those based on the classical sinc method [15].

Tables 5 and 6 list the exact solutions $\mu_{k}$ for two choices of $m$ and $\theta$ at $N=20$ and different values of $\varepsilon$. It is indicated

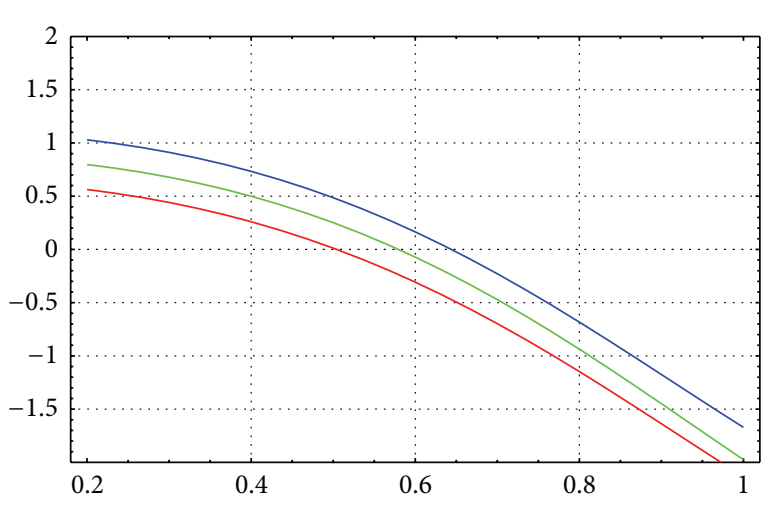

FIGURE 1: $a_{+}, \Gamma(\mu)$, and $a_{-}$with $N=20, m=8, \theta=1 / 6$, and $\varepsilon=10^{-5}$.

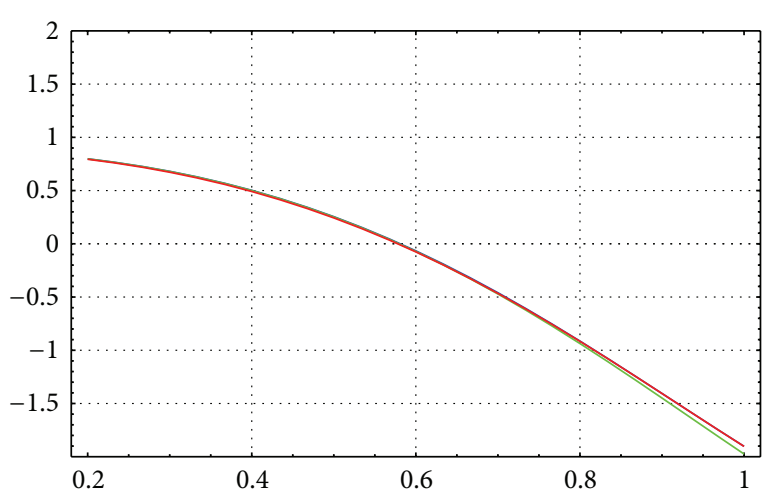

FIGURE 2: $a_{+}, \Gamma(\mu)$, and $a_{-}$with $N=20, m=8, \theta=1 / 6$, and $\varepsilon=$ $10^{-10}$.

that the solutions $\mu_{k}$ are all inside the interval $\left[a_{-}, a_{+}\right]$for all values of $\varepsilon$.

For $N=20, m=8$, and $\theta=1 / 6$, Figures 1 and 2 illustrate the enclosure intervals dominating $\mu_{1}$ for $\varepsilon=10^{-5}$ and $\varepsilon=10^{-10}$, respectively. The middle curve represents $\Gamma(\mu)$, while the upper and lower curves represent the curves of $a_{+}(\mu), a_{-}(\mu)$, respectively. We notice that when $\varepsilon=10^{-10}$ all 
TABLE 3: Absolute errors $\left|\mu_{k}-\mu_{k, N}\right|$ for $N=20, m=8$, and $\theta=1 / 6$.

\begin{tabular}{lccccc}
\hline$\mu_{k}$ & $\mu_{1}$ & $\mu_{2}$ & $\mu_{3}$ & $\mu_{4}$ & $\mu_{5}$ \\
\hline$E_{S}[15]$ & $1.442 \times 10^{-8}$ & $3.757 \times 10^{-9}$ & $5.972 \times 10^{-9}$ & $19.358 \times 10^{-10}$ & $4.538 \times 10^{-9}$ \\
$E_{H}$ & $6.625 \times 10^{-12}$ & $1.954 \times 10^{-13}$ & $5.138 \times 10^{-13}$ & $8.882 \times 10^{-15}$ & $1.608 \times 10^{-13}$ \\
\hline
\end{tabular}

TABLE 4: Absolute errors $\left|\mu_{k}-\mu_{k, N}\right|$ for $N=20, m=12$, and $\theta=1 / 4$.

\begin{tabular}{|c|c|c|c|c|c|}
\hline$\underline{\mu_{k}}$ & $\mu_{1}$ & $\mu_{2}$ & $\mu_{3}$ & $\mu_{4}$ & $\mu_{5}$ \\
\hline$E_{S}[15]$ & $6.332 \times 10^{-13}$ & $1.206 \times 10^{-12}$ & $1.207 \times 10^{-12}$ & $6.397 \times 10^{-12}$ & $1.025 \times 10^{-11}$ \\
\hline$E_{H}$ & $2.3 \times 10^{-18}$ & $2.8 \times 10^{-18}$ & $1.5 \times 10^{-18}$ & $1.01 \times 10^{-17}$ & $2.3 \times 10^{-18}$ \\
\hline
\end{tabular}

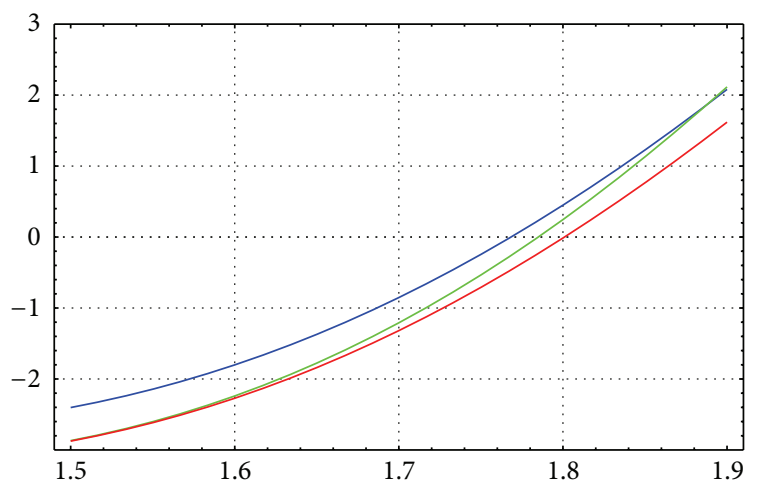

Figure $3: a_{+}, \Gamma(\mu)$, and $a_{-}$with $N=20, m=8, \theta=1 / 6$, and $\varepsilon=10^{-5}$.

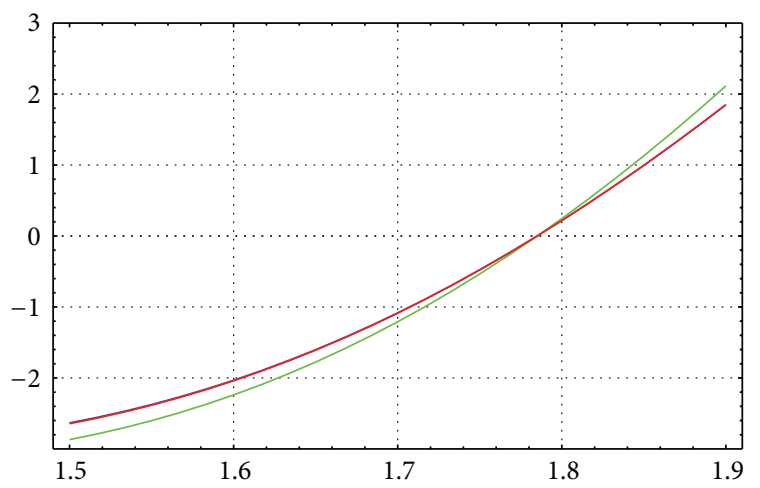

FIgURE 4: $a_{+}, \Gamma(\mu)$, and $a_{-}$with $N=20, m=8, \theta=1 / 6$, and $\varepsilon=$ $10^{-10}$.

three curves are almost identical. Similarly, Figures 3 and 4 illustrate the enclosure intervals dominating $\mu_{2}$ for $\varepsilon=10^{-5}$, $\varepsilon=10^{-10}$, respectively.

As in Table 6 , for $N=20, m=12$, and $\theta=1 / 4$, Figures 5 and 6 illustrate the enclosure intervals dominating $\mu_{3}$ for $\varepsilon=10^{-5}$ and $\varepsilon=10^{-10}$, respectively, and Figures 7 and 8 illustrate the enclosure intervals dominating $\mu_{4}$ for $\varepsilon=10^{-5}$, $\varepsilon=10^{-10}$, respectively.

Example 2. Consider the boundary value problem

$$
\begin{array}{r}
-\mathfrak{y}^{\prime \prime}(x, \mu)+q(x) \mathfrak{y}(x, \mu)=\mu^{2} \mathfrak{y}(x, \mu), \\
x \in[-1,0) \cup(0,1],
\end{array}
$$

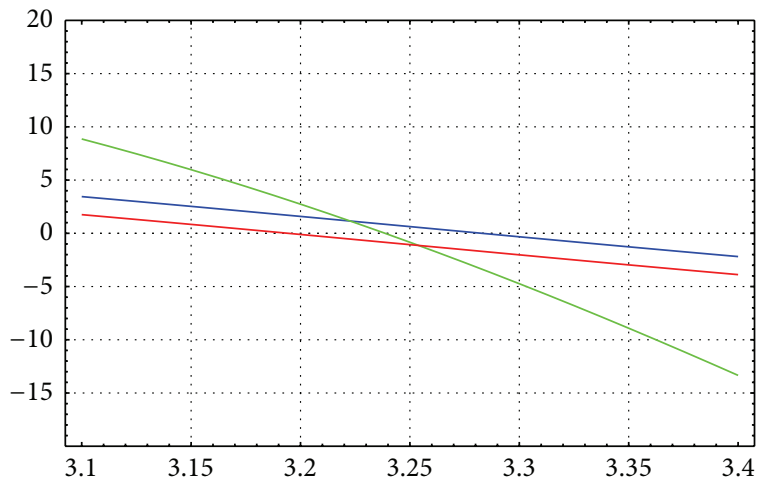

Figure 5: $a_{+}, \Gamma(\mu)$, and $a_{-}$with $N=20, m=12, \theta=1 / 4$, and $\varepsilon=10^{-5}$.

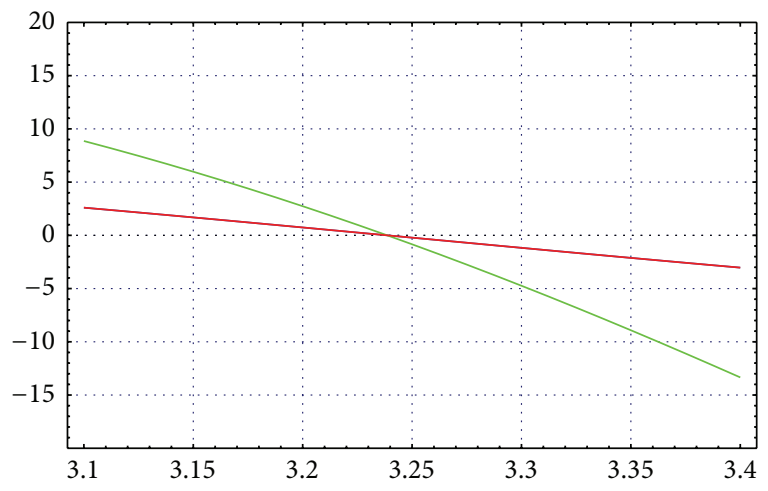

Figure 6: $a_{+}, \Gamma(\mu)$, and $a_{-}$with $N=20, m=12, \theta=1 / 4$, and $\varepsilon=10^{-10}$.

$$
\begin{gathered}
\mathfrak{y}(-1, \mu)+\mu^{2} \mathfrak{y}^{\prime}(-1, \mu)=0, \\
\mathfrak{y}(1, \mu)+\mu^{2} \mathfrak{y}^{\prime}(1, \mu)=0, \\
\mathfrak{y}\left(0^{-}, \mu\right)-\mathfrak{y}\left(0^{+}, \mu\right)=0, \quad \mathfrak{y}^{\prime}\left(0^{-}, \mu\right)-\mathfrak{y}^{\prime}\left(0^{+}, \mu\right)=0,
\end{gathered}
$$

where $\alpha_{1}=\beta_{1}=1, \alpha_{2}^{\prime}=\beta_{2}^{\prime}=-1, \beta_{1}^{\prime}=\beta_{2}=\alpha_{2}=\alpha_{1}^{\prime}=0$, $\gamma_{1}=\delta_{1}=3, \gamma_{2}=\delta_{2}=1 / 3$, and

$$
q(x)= \begin{cases}-2, & x \in[-1,0) \\ x, & x \in(0,1]\end{cases}
$$


TABLE 5: For $N=20, m=8$, and $\theta=1 / 6$, the exact solutions $\mu_{k}$ are all inside the interval $\left[a_{-}, a_{+}\right]$for different values of $\varepsilon$.

\begin{tabular}{lccc}
\hline$\mu_{k}$ & Exact $\mu_{k}$ & {$\left[a_{-}, a_{+}\right], \varepsilon=10^{-5}$} & {$\left[a_{-}, a_{+}\right], \varepsilon=10^{-10}$} \\
\hline$\mu_{1}$ & 0.579603114810978 & {$[0.504287,0.644005]$} & {$[0.579603,0.581089]$} \\
$\mu_{2}$ & 1.7849838948888357 & {$[1.76837,1.80095]$} & {$[1.78494,1.78503]$} \\
$\mu_{3}$ & 3.238647349751419 & {$[3.18936,3.28517]$} & {$[3.2375,3.23979]$} \\
$\mu_{4}$ & 4.770562335590527 & {$[4.74591,4.79495]$} & {$[4.77054,4.77059]$} \\
$\mu_{5}$ & 6.3257043646722515 & {$[6.30433,6.34704]$} & {$[6.32536,6.32605]$} \\
\hline
\end{tabular}

$\mathscr{E}_{3}\left(\mathscr{F}_{\theta, m}\right)=2.61231 \times 10^{11}, \mathscr{E}_{2}\left(\mathscr{F}_{\theta, m}\right)=4.67787 \times 10^{10}, \alpha=1$, and $\mathscr{M}_{\mathscr{F}_{\theta, m}}=5.31641 \times 10^{9}$.

TABLE 6: For $N=20, m=12$; and $\theta=1 / 4, \mu_{k}$ are all inside the interval $\left[a_{-}, a_{+}\right]$for different values of $\varepsilon$.

\begin{tabular}{lcrr}
\hline$\mu_{k}$ & Exact $\mu_{k}$ & {$\left[a_{-}, a_{+}\right], \varepsilon=10^{-5}$} & {$\left[a_{-}, a_{+}\right], \varepsilon=10^{-10}$} \\
\hline$\mu_{1}$ & 0.57960311481097786078 & {$[0.130168,0.796074]$} & {$[0.579579,0.579627]$} \\
$\mu_{2}$ & 1.784983894888883561160 & {$[1.699182,1.859482]$} & {$[1.784963,1.785004]$} \\
$\mu_{3}$ & 3.23864734975141899542 & {$[3.194355,3.282835]$} & {$[3.238636,3.238658]$} \\
$\mu_{4}$ & 4.77056233559052749760 & {$[4.698242,4.851169]$} & {$[4.770489,4.770635]$} \\
$\mu_{5}$ & 6.32570436467225155153 & {$[6.287476,6.368771]$} & {$[6.325701,6.325707]$} \\
$\mathscr{E}_{7}\left(\mathscr{F}_{\theta, m}\right)=2.25863 \times 10^{13}, \mathscr{E}_{6}\left(\mathscr{F}_{\theta, m}\right)=5.91004 \times 10^{12}, \alpha=1$, and $\mathscr{M}_{\mathscr{F}_{\theta, m}}=2.11965 \times 10^{9}$.
\end{tabular}

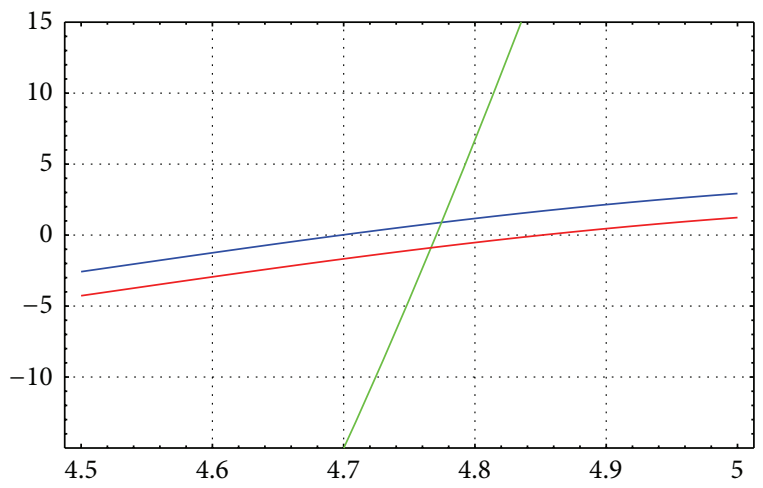

FIGURE 7: $a_{+}, \Gamma(\mu)$, and $a_{-}$with $N=20, m=12, \theta=1 / 4$, and $\varepsilon=10^{-5}$.

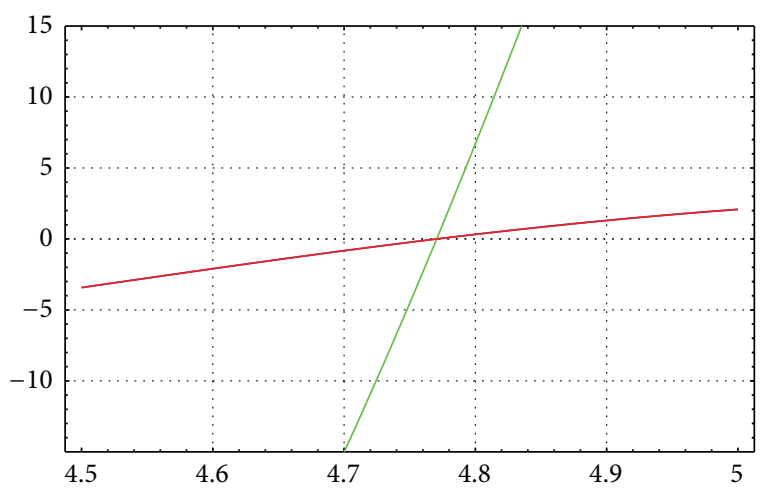

Figure $8: a_{+}, \Gamma(\mu)$, and $a_{-}$with $N=20, m=12, \theta=1 / 4$, and $\varepsilon=10^{-10}$.

The function $\mathscr{K}(\mu)$ will be

$$
\mathscr{K}(\mu)=\frac{\left(1+\mu^{6}\right) \sin 2 \mu}{\mu} .
$$

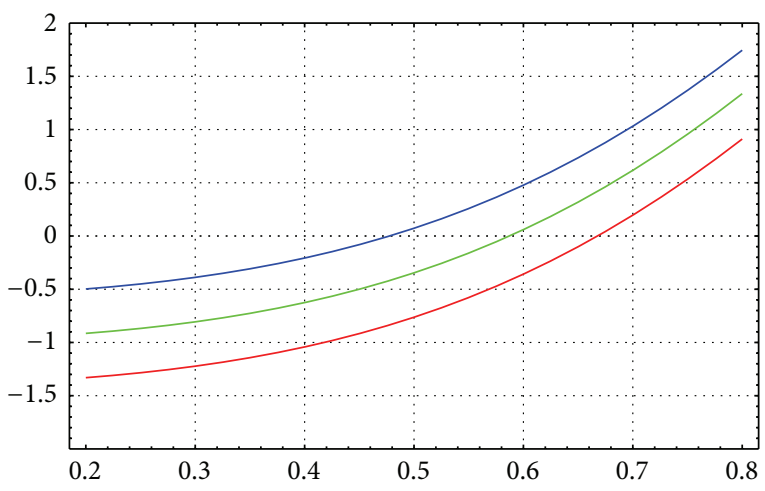

Figure 9: $a_{+}, \Gamma(\mu)$, and $a_{-}$with $N=40, m=12, \theta=1 / 14$, and $\varepsilon=10^{-6}$.

The application of Hermite interpolations method and sinc method [15] to this problem and the effect of $\theta$ and $m$ at $N=40$ are indicated in Tables 7 and 8. In Tables 9 and 10, we display the maximum absolute error of $\mu_{k}-\mu_{k, N}$, using Hermite interpolations method and sinc method [15] with various choices of $\theta$ and $m$ at $N=40$. Form these tables, it is shown that the proposed methods are significantly more accurate than those based on the classical sinc method [15].

Tables 11 and 12 list the exact solutions $\mu_{k}$ for two choices of $m$ and $\theta$ at $N=40$ and different values of $\varepsilon$. It is indicated that the solutions $\mu_{k}$ are all inside the interval $\left[a_{-}, a_{+}\right]$for all values of $\varepsilon$.

For $N=40, m=12$, and $\theta=1 / 14$, Figures 9 and 10 illustrate the enclosure intervals dominating $\mu_{1}$ for $\varepsilon=$ $10^{-6}$ and $\varepsilon=10^{-12}$, respectively. Similarly, Figures 11 and 12 illustrate the enclosure intervals dominating $\mu_{2}$ for $\varepsilon=10^{-6}$, $\varepsilon=10^{-12}$, respectively.

For $N=40, m=16$, and $\theta=1 / 12$, Figures 13 and 14 illustrate the enclosure intervals dominating $\mu_{1}$ for $\varepsilon=10^{-7}$ and $\varepsilon=10^{-12}$, respectively, and Figures 15 and 16 illustrate 
TABLE 7: Comparing the exact, sinc, and Hermite solutions at $N=40, m=12$, and $\theta=1 / 14$.

\begin{tabular}{lccc}
\hline$\mu_{k}$ & Sinc $\mu_{k, N}[15]$ & Exact $\mu_{k}$ & Hermite $\mu_{k, N}$ \\
\hline$\mu_{1}$ & 0.58718720635351373438 & 0.58718716772603949302 & 0.58718716772603439313 \\
$\mu_{2}$ & 1.67733213643112965108 & 1.677332124049777904702 & 1.67733212404977962102 \\
$\mu_{3}$ & 3.05318928070135885375 & 3.05318927948461569256 & 3.05318927948461567248 \\
$\mu_{4}$ & 4.64836948022942956741 & 4.64836948049457049728 & 4.64836948049456789884 \\
$\mu_{5}$ & 6.22695152015827473816 & 6.22695152029019996978 & 6.22695152029019996831 \\
\hline
\end{tabular}

TABLE 8: Comparing the exact, sinc, and Hermite solutions at $N=40, m=16$, and $\theta=1 / 12$.

\begin{tabular}{lccc}
\hline$\mu_{k}$ & Sinc $\mu_{k, N}[15]$ & Exact $\mu_{k}$ & Hermite $\mu_{k, N}$ \\
\hline$\mu_{1}$ & 0.58718716772553041066238963 & 0.58718716772603949302708573 & 0.58718716772603949300999675 \\
$\mu_{2}$ & 1.67733212404966397991332993 & 1.67733212404977904702706192 & 1.67733212404977904702798867 \\
$\mu_{3}$ & 3.05318927948463571892007113 & 3.05318927948461569256662121 & 3.05318927948461569256652760 \\
$\mu_{4}$ & 4.648369480494569410072581778 & 4.64836948049456789592198501 & 4.64836948049456789592198641 \\
$\mu_{5}$ & 6.22695152029019694414158617 & 6.2269515202901999697647050 & 6.226951520290199969764695999 \\
\hline
\end{tabular}

TABLE 9: Absolute errors $\left|\mu_{k}-\mu_{k, N}\right|$ for $N=40, m=12$, and $\theta=1 / 14$.

\begin{tabular}{lccccc}
\hline$\mu_{k}$ & $\mu_{1}$ & $\mu_{2}$ & $\mu_{3}$ & $\mu_{4}$ & $\mu_{5}$ \\
\hline$E_{S}[15]$ & $3.863 \times 10^{-8}$ & $1.238 \times 10^{-8}$ & $1.217 \times 10^{-9}$ & $2.651 \times 10^{-10}$ & $1.319 \times 10^{-10}$ \\
$E_{H}$ & $5.099 \times 10^{-15}$ & $5.739 \times 10^{-16}$ & $2.009 \times 10^{-17}$ & $2.598 \times 10^{-15}$ & $1.467 \times 10^{-18}$ \\
\hline
\end{tabular}

TABLE 10: Absolute errors $\left|\mu_{k}-\mu_{k, N}\right|$ for $N=40, m=16$, and $\theta=1 / 12$.

\begin{tabular}{lccccc}
\hline$\mu_{k}$ & $\mu_{1}$ & $\mu_{2}$ & $\mu_{3}$ & $\mu_{4}$ & $\mu_{5}$ \\
\hline$E_{S}[15]$ & $5.091 \times 10^{-13}$ & $1.1506 \times 10^{-13}$ & $2.003 \times 10^{-14}$ & $1.514 \times 10^{-15}$ & $3.025 \times 10^{-15}$ \\
$E_{H}$ & $1.708 \times 10^{-20}$ & $9.267 \times 10^{-22}$ & $9.361 \times 10^{-23}$ & $1.403 \times 10^{-24}$ & $9.010 \times 10^{-24}$ \\
\hline
\end{tabular}

TABLE 11: For $N=40, m=12$, and $\theta=1 / 14, \mu_{k}$ are all inside the interval $\left[a_{-}, a_{+}\right]$for different values of $\varepsilon$.

\begin{tabular}{lcrr}
\hline$\mu_{k}$ & Exact $\mu_{k}$ & {$\left[a_{-}, a_{+}\right], \varepsilon=10^{-6}$} & {$\left[a_{-}, a_{+}\right], \varepsilon=10^{-12}$} \\
\hline$\mu_{1}$ & 0.58718716772603949302 & {$[0.477526,0.667764]$} & {$[0.586774,0.587599]$} \\
$\mu_{2}$ & 1.67733212404977904702 & {$[1.666867,1.687341]$} & {$[1.677285,1.677378]$} \\
$\mu_{3}$ & 3.05318927948461569256 & {$[3.046882,3.059372]$} & {$[3.053187,3.053190]$} \\
$\mu_{4}$ & 4.64836948049457049728 & {$[4.647491,4.649246]$} & {$[4.64836924,4.64836971]$} \\
$\mu_{5}$ & 6.22695152029019996978 & {$[6.226707,6.227195]$} & {$[6.22695140,6.22695163]$} \\
\hline $\mathscr{E}_{7}\left(\mathscr{F}_{\theta, m}\right)=6.29794 \times 10^{19}, \mathscr{E}_{6}\left(\mathscr{F}_{\theta, m}\right)=4.70791 \times 10^{18}, \alpha=1$, and $\mathscr{M}_{\mathscr{F}_{\theta, m}}=4.71851 \times 10^{12}$.
\end{tabular}

TABLE 12: For $N=40, m=16$, and $\theta=1 / 12, \mu_{k}$ are all inside the interval $\left[a_{-}, a_{+}\right]$for different values of $\varepsilon$.

\begin{tabular}{lccc}
\hline$\mu_{k}$ & Exact $\mu_{k}$ & {$\left[a_{-}, a_{+}\right], \varepsilon=10^{-7}$} & {$\left[a_{-}, a_{+}\right], \varepsilon=10^{-12}$} \\
\hline$\mu_{1}$ & 0.58718716772603949302708573 & {$[0.554227,0.616993]$} & {$[0.587165,0.587209]$} \\
$\mu_{2}$ & 1.67733212404977904702706192 & {$[1.648191,1.703225]$} & {$[1.6773305,1.6773337]$} \\
$\mu_{3}$ & 3.05318927948461569256662121 & {$[3.050775,3.055585]$} & {$[3.05318912,3.05318943]$} \\
$\mu_{4}$ & 4.64836948049456789592198501 & {$[4.647994,4.648743]$} & {$[4.648369468,4.648369492]$} \\
$\mu_{5}$ & 6.2269515202901999697647050 & {$[6.226831,6.227072]$} & {$[6.226951509,6.226951531]$} \\
\hline $\mathscr{E}_{11}\left(\mathscr{F}_{\theta, m}\right)=1.67235 \times 10^{24}, \mathscr{E}_{10}\left(\mathscr{F}_{\theta, m}\right)=1.44089 \times 10^{23}, \alpha=1$, and $\mathscr{M}_{\mathscr{F}_{\theta, m}}=1.28771 \times 10^{13}$. &
\end{tabular}

TABLE 13: Comparing the exact, sinc, and Hermite solutions at $N=40, m=2$.

\begin{tabular}{cccccc}
\hline$\mu_{k}$ & Sinc $\mu_{k, N}[9]$ & Exact $\mu_{k}$ & Hermite $\mu_{k, N}$ & $E_{S}$ & $E_{H}$ \\
\hline$\mu_{1}$ & 3.119437080035764 & 3.1194369008225 & 3.1194368826279857 & $1.79213 \times 10^{-7}$ & $1.81945 \times 10^{-8}$ \\
$\mu_{2}$ & 9.421424381799804 & 9.421428536270897 & 9.421428567680044 & $4.15447 \times 10^{-6}$ & $3.14091 \times 10^{-8}$ \\
$\mu_{3}$ & 12.565198874263179 & 12.565194405126995 & 12.56519440514131 & $4.46914 \times 10^{-6}$ & $1.43157 \times 10^{-11}$ \\
\hline
\end{tabular}




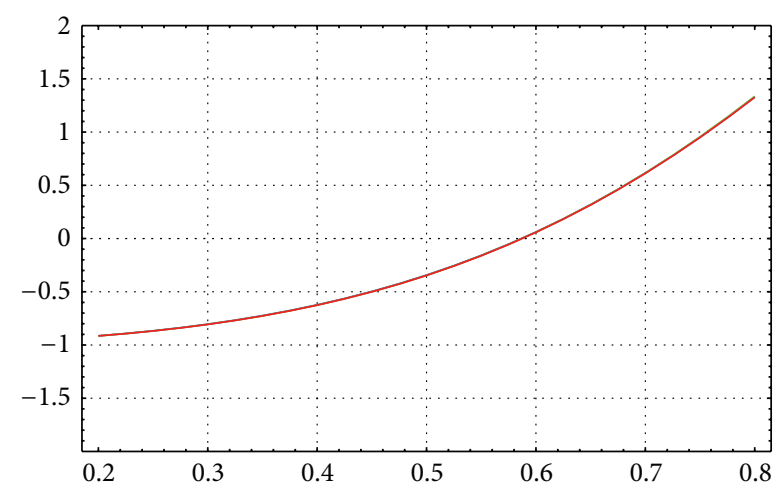

FIgURE 10: $a_{+}, \Gamma(\mu)$, and $a_{-}$with $N=40, m=12, \theta=1 / 14$, and $\varepsilon=10^{-12}$.

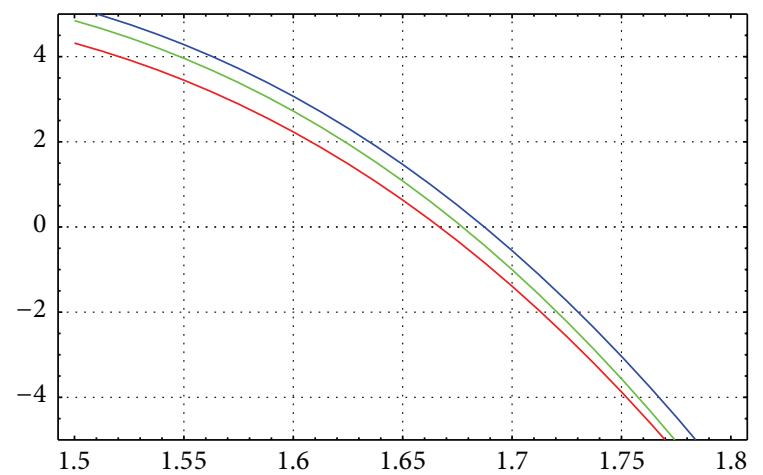

Figure 11: $a_{+}, \Gamma(\mu)$, and $a_{-}$with $N=40, m=12, \theta=1 / 14$, and $\varepsilon=10^{-6}$.

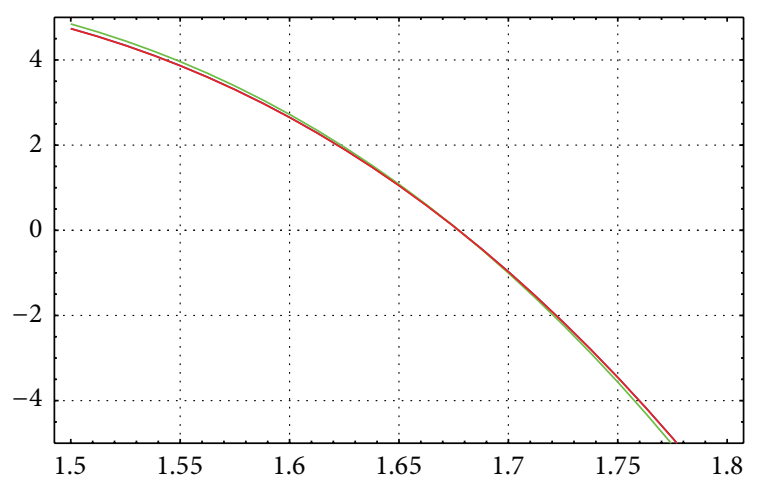

FIGURE 12: $a_{+}, \Gamma(\mu)$, and $a_{-}$with $N=40, m=12, \theta=1 / 14$, and $\varepsilon=10^{-12}$.

the enclosure intervals dominating $\mu_{2}$ for $\varepsilon=10^{-7}, \varepsilon=10^{-12}$, respectively.

Example 3. Consider the continuous boundary value problem [9]

$$
\begin{gathered}
-\mathfrak{y}^{\prime \prime}(x, \mu)=\mu^{2} \mathfrak{y}(x, \mu), \quad x \in[0,1], \\
-\mathfrak{y}(0, \mu)=\left(\mu^{2}+d\right) \mathfrak{y}^{\prime}(0, \mu), \quad \mathfrak{y}(1, \mu)=\mu^{2} \mathfrak{y}^{\prime}(1, \mu),
\end{gathered}
$$

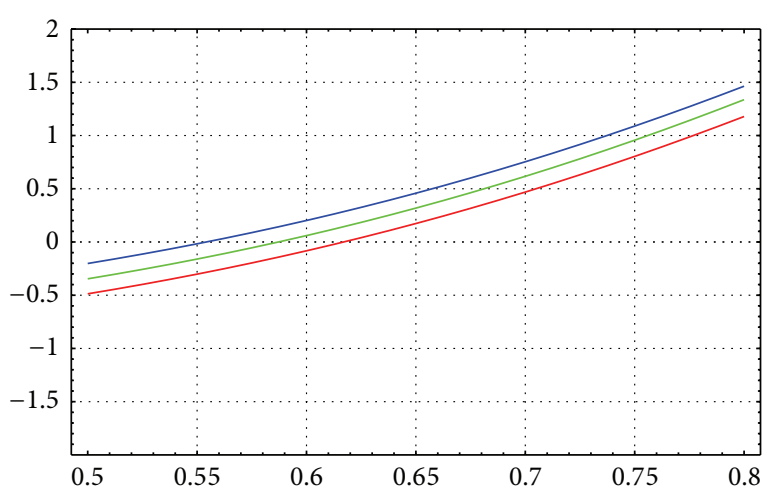

FIGURE 13: $a_{+}, \Gamma(\mu)$, and $a_{-}$with $N=40, m=16, \theta=1 / 12$, and $\varepsilon=10^{-7}$.

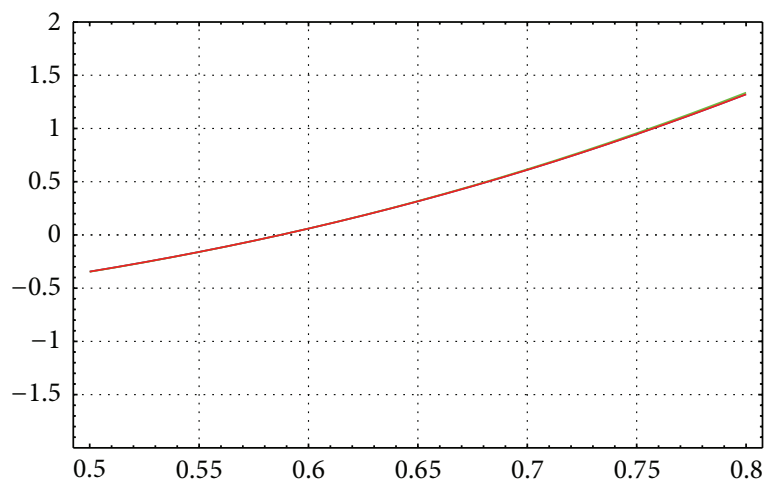

FIGURE 14: $a_{+}, \Gamma(\mu)$, and $a_{-}$with $N=40, m=16, \theta=1 / 12$, and $\varepsilon=10^{-12}$.

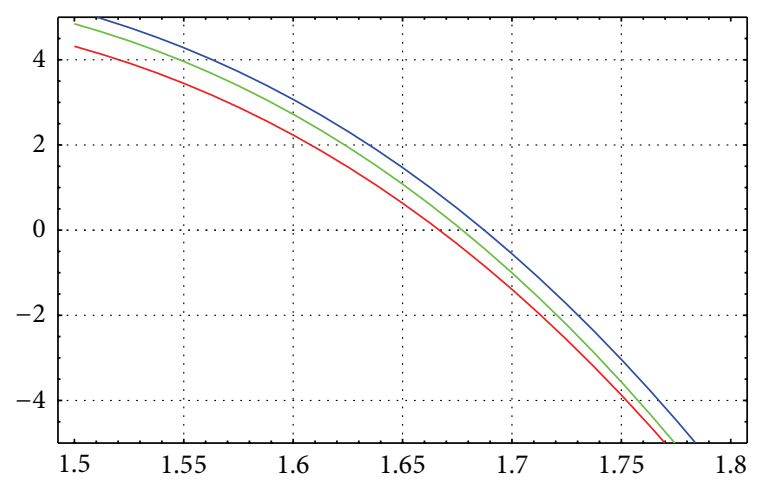

FIGURE 15: $a_{+}, \Gamma(\mu)$, and $a_{-}$with $N=40, m=16, \theta=1 / 12$, and $\varepsilon=10^{-7}$.

where $d=-4 \pi^{2}, \beta_{1}=\beta_{2}^{\prime}=1, \alpha_{2}=d, \alpha_{1}=\beta_{2}=\beta_{1}^{\prime}=0$, $\alpha_{2}=d$, and $\alpha_{2}^{\prime}=-1$. The exact characteristic function is

$$
\Gamma(\mu)=\left(1+4 \pi^{2} \mu^{4}-\mu^{6}\right) \frac{\sin \mu}{\mu}-\left(2 \mu^{2}-4 \pi^{2}\right) \cos \mu,
$$

where zero is not an eigenvalue. The application of Hermite interpolations method and sinc method [9] to this problem is indicated in Table 13. From this table, it is shown that the proposed method is significantly more accurate than that based on the sinc method [9]. 


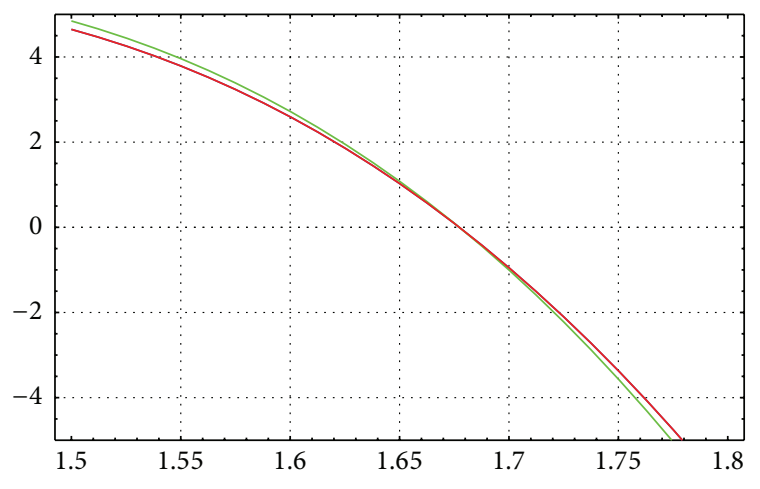

Figure 16: $a_{+}, \Gamma(\mu)$, and $a_{-}$with $N=40, m=16, \theta=1 / 12$, and $\varepsilon=10^{-12}$.

\section{Acknowledgments}

This work was funded by the Deanship of Scientific Research (DSR), King Abdulaziz University, Jeddah, under Grant no. 130-065-D1433. The authors, therefore, acknowledge with thanks DSR technical and financial support.

\section{References}

[1] E. H. Doha, A. H. Bhrawy, and R. M. Hafez, "A Jacobi-Jacobi dual-Petrov-Galerkin method for third- and fifth-order differential equations," Mathematical and Computer Modelling, vol. 53, no. 9-10, pp. 1820-1832, 2011.

[2] E. H. Doha, A. H. Bhrawy, and R. M. Hafez, "A Jacobi dualPetrov-Galerkin method for solving some odd-order ordinary differential equations," Abstract and Applied Analysis, vol. 2011, Article ID 947230, 21 pages, 2011.

[3] E. Tohidi, A. H. Bhrawy, and K. Erfani, "A collocation method based on Bernoulli operational matrix for numerical solution of generalized pantograph equation," Applied Mathematical Modelling, vol. 37, no. 6, pp. 4283-4294, 2013.

[4] A. H. Bhrawy, A. S. Alofi, and S. I. El-Soubhy, "An extension of the Legendre-Galerkin method for solving sixth-order differential equations with variable polynomial coefficients," Mathematical Problems in Engineering, vol. 2012, Article ID 896575, 13 pages, 2012.

[5] A. H. Bhrawy, "A Jacobi-Gauss-Lobatto collocation method for solving generalized Fitzhugh-Nagumo equation with timedependent coefficients," Applied Mathematics and Computation, vol. 222, pp. 255-264, 2013.

[6] A. H. Bhrawy, M. M. Tharwat, and A. Al-Fhaid, "Numerical algorithms for computing eigenvalues of discontinuous Dirac system using sinc-Gaussian method," Abstract and Applied Analysis, vol. 2012, Article ID 925134, 13 pages, 2012.

[7] A. Imani, A. Aminataei, and A. Imani, "Collocation method via Jacobi polynomials for solving nonlinear ordinary differential equations," International Journal of Mathematics and Mathematical Sciences, vol. 2011, Article ID 673085, 11 pages, 2011.

[8] A. Boumenir and B. Chanane, "Eigenvalues of Sturm-Liouville systems using sampling theory," Applied Analysis, vol. 62, pp. 323-334, 1996.

[9] B. Chanane, "Computation of the eigenvalues of SturmLiouville problems with parameter dependent boundary conditions using the regularized sampling method," Mathematics of Computation, vol. 74, no. 252, pp. 1793-1801, 2005.
[10] B. Chanane, "Computing the spectrum of non-self-adjoint Sturm-Liouville problems with parameter-dependent boundary conditions," Journal of Computational and Applied Mathematics, vol. 206, no. 1, pp. 229-237, 2007.

[11] B. Chanane, "Computing the eigenvalues of singular SturmLiouville problems using the regularized sampling method," Applied Mathematics and Computation, vol. 184, no. 2, pp. 972978, 2007.

[12] B. Chanane, "Eigenvalues of Sturm-Liouville problems with discontinuity conditions inside a finite interval," Applied Mathematics and Computation, vol. 188, no. 2, pp. 1725-1732, 2007.

[13] B. Chanane, "Sturm-Liouville problems with impulse effects," Applied Mathematics and Computation, vol. 190, no. 1, pp. 610626, 2007.

[14] M. M. Tharwat, "Discontinuous Sturm-Liouville problems and associated sampling theories," Abstract and Applied Analysis, vol. 2011, Article ID 610232, 30 pages, 2011.

[15] M. M. Tharwat, A. H. Bhrawy, and A. Yildirim, "Numerical computation of eigenvalues of discontinuous Sturm-Liouville problems with parameter dependent boundary conditions using sinc method," Numerical Algorithms, vol. 63, no. 1, pp. 2748, 2013.

[16] V. Kotelnikov, "On the carrying capacity of the "ether" and wire in telecommunications," in Proceedings of the 1st all union conference on questions of communications, Izd. Red. Upr. Svyazi RKKA, Moscow, Russia, 1933.

[17] C. E. Shannon, "Communication in the presence of noise," Proceedings of the IEEE, vol. 37, pp. 10-21, 1949.

[18] E. Whittaker, "On the functions which are represented by the expansion of the interpolation theory," Proceedings of the Royal Society of Edinburgh A, vol. 35, pp. 181-194, 1915.

[19] G. R. Grozev and Q. I. Rahman, "Reconstruction of entire functions from irregularly spaced sample points," Canadian Journal of Mathematics, vol. 48, no. 4, pp. 777-793, 1996.

[20] J. R. Higgins, G. Schmeisser, and J. J. Voss, "The sampling theorem and several equivalent results in analysis," Journal of Computational Analysis and Applications, vol. 2, no. 4, pp. 333-371, 2000.

[21] G. Hinsen, "Irregular sampling of bandlimited $L^{p}$-functions," Journal of Approximation Theory, vol. 72, no. 3, pp. 346-364, 1993.

[22] D. Jagerman and L. Fogel, "Some general aspects of the sampling theorem," IRE Transactions on Information Theory, vol. 2, pp. 139-146, 1956.

[23] M. H. Annaby and R. M. Asharabi, "Error analysis associated with uniform Hermite interpolations of bandlimited functions," Journal of the Korean Mathematical Society, vol. 47, no. 6, pp. 1299-1316, 2010.

[24] J. R. Higgins, Sampling Theory in Fourier and Signal Analysis: Foundations, Oxford University Press, Oxford, UK, 1996.

[25] P. L. Butzer, J. R. Higgins, and R. L. Stens, "Sampling theory of signal analysis," in Development of Mathematics 1950-2000, pp. 193-234, Birkhäuser, Basel, Switzerland, 2000.

[26] P. L. Butzer, G. Schmeisser, and R. L. Stens, "An introduction to sampling analysis," in Nonuniform Sampling, F. Marvasti, Ed., pp. 17-121, Kluwer, New York, NY, USA, 2001.

[27] A. Boumenir, "Higher approximation of eigenvalues by the sampling method," BIT, vol. 40, no. 2, pp. 215-225, 2000.

[28] M. M. Tharwat, A. H. Bhrawy, and A. Yildirim, "Numerical computation of the eigenvalues of a discontinuous Dirac system using the sinc method with error analysis," International Journal of Computer Mathematics, vol. 89, no. 15, pp. 2061-2080, 2012. 
[29] J. Lund and K. L. Bowers, Sinc Methods for Quadrature and Differential Equations, Society for Industrial and Applied Mathematics (SIAM), Philadelphia, Pa, USA, 1992.

[30] F. Stenger, "Numerical methods based on Whittaker cardinal, or sinc functions," SIAM Review, vol. 23, no. 2, pp. 165-224, 1981.

[31] F. Stenger, Numerical Methods Based on Sinc and Analytic Functions, vol. 20, Springer, New York, NY, USA, 1993.

[32] P. L. Butzer, W. Splettstösser, and R. L. Stens, “The sampling theorem and linear prediction in signal analysis," Jahresbericht der Deutschen Mathematiker-Vereinigung, vol. 90, no. 1, p. 70, 1988.

[33] D. Jagerman, "Bounds for truncation error of the sampling expansion," SIAM Journal on Applied Mathematics, vol. 14, pp. 714-723, 1966.

[34] M. M. Tharwat and A. H. Bhrawy, "Computation of eigenvalues of discontinuous Dirac system using Hermite interpolation technique," Advances in Difference Equations, vol. 2012, article 59, 2012

[35] M. M. Tharwat, A. H. Bhrawy, and A. S. Alofi, "Approximation of eigenvalues of discontinuous Sturm-Liouville problems with eigenparameter in all boundary conditions," Boundary Value Problems, vol. 2013, article 132, 2013.

[36] M. Kadakal and O. S. Mukhtarov, "Discontinuous SturmLiouville problems containing eigenparameter in the boundary conditions," Acta Mathematica Sinica, vol. 22, no. 5, pp. 15191528, 2006.

[37] O. S. Mukhtarov, M. Kadakal, and N. Altinisik, "Eigenvalues and eigenfunctions of discontinuous Sturm-Liouville problems with eigenparameter in the boundary conditions," Indian Journal of Pure and Applied Mathematics, vol. 34, no. 3, pp. 501-516, 2003.

[38] M. M. Tharwat, A. Yildirim, and A. H. Bhrawy, "Sampling of discontinuous Dirac systems," Numerical Functional Analysis and Optimization, vol. 34, no. 3, pp. 323-348, 2013. 


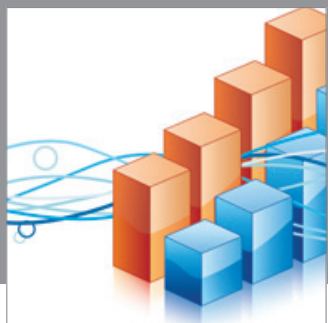

Advances in

Operations Research

mansans

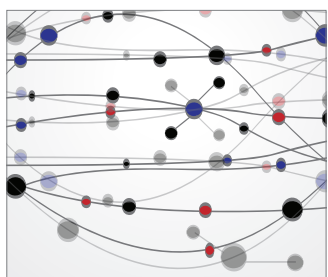

The Scientific World Journal
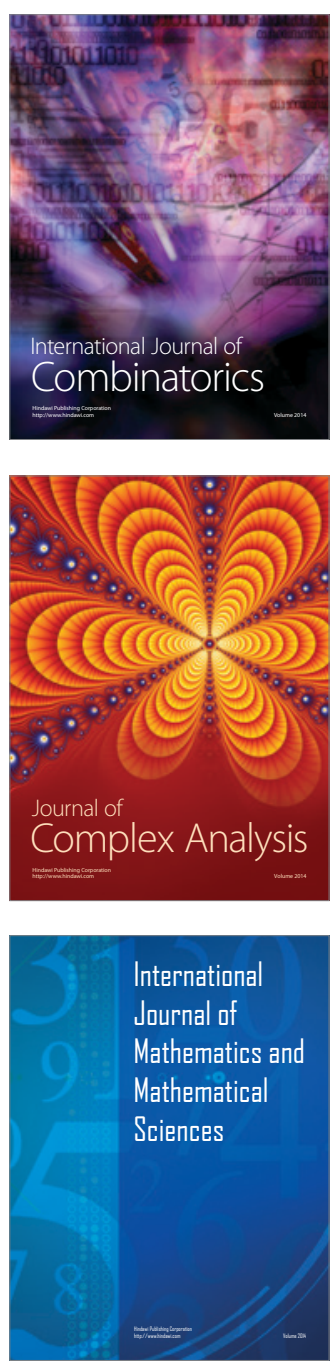
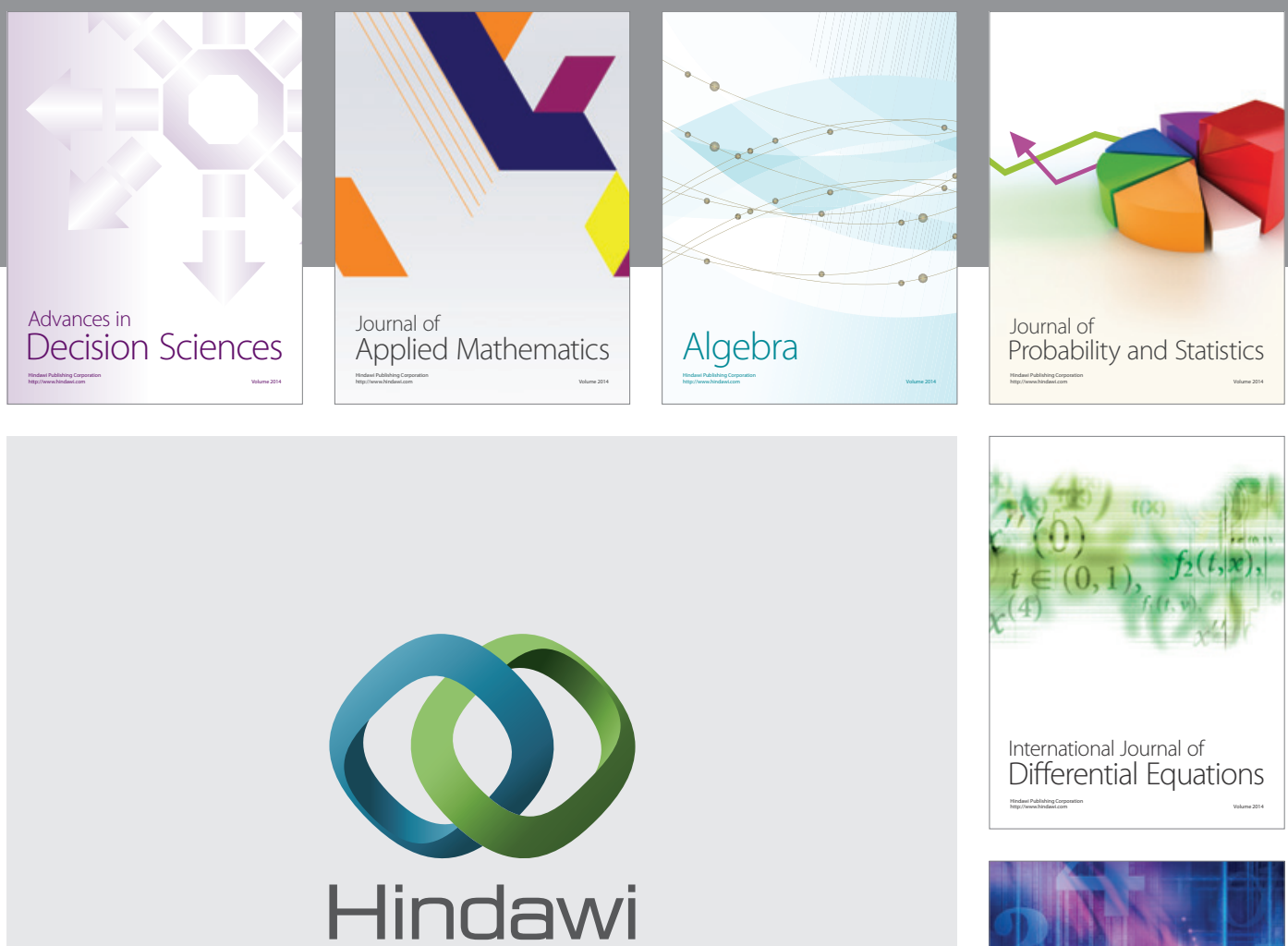

Submit your manuscripts at http://www.hindawi.com
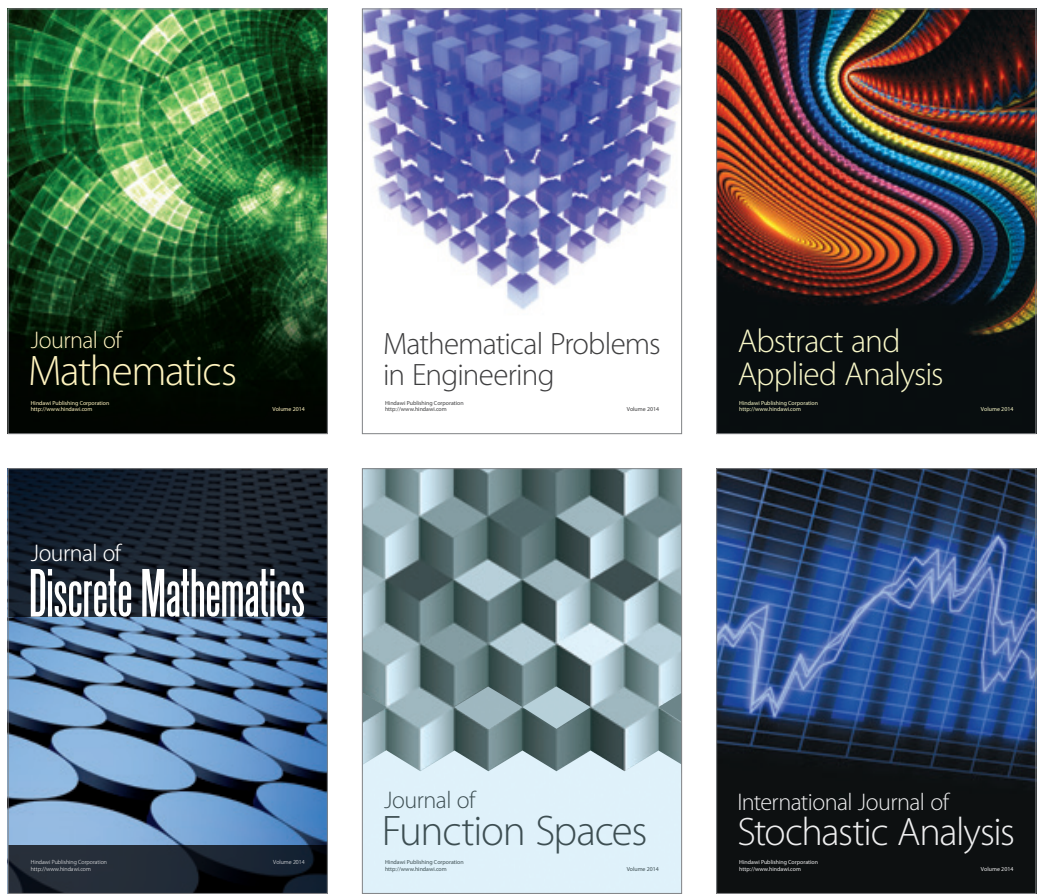

Journal of

Function Spaces

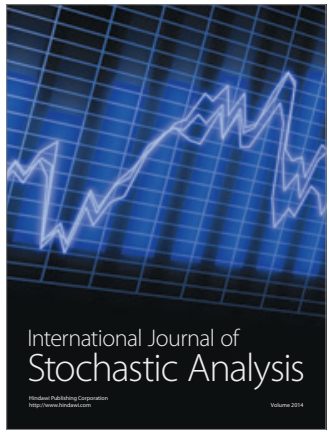

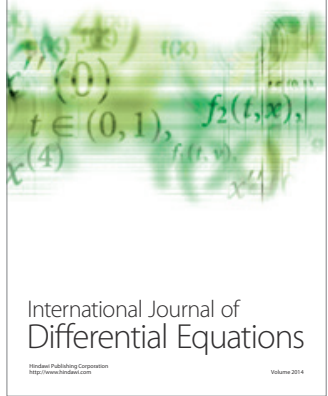
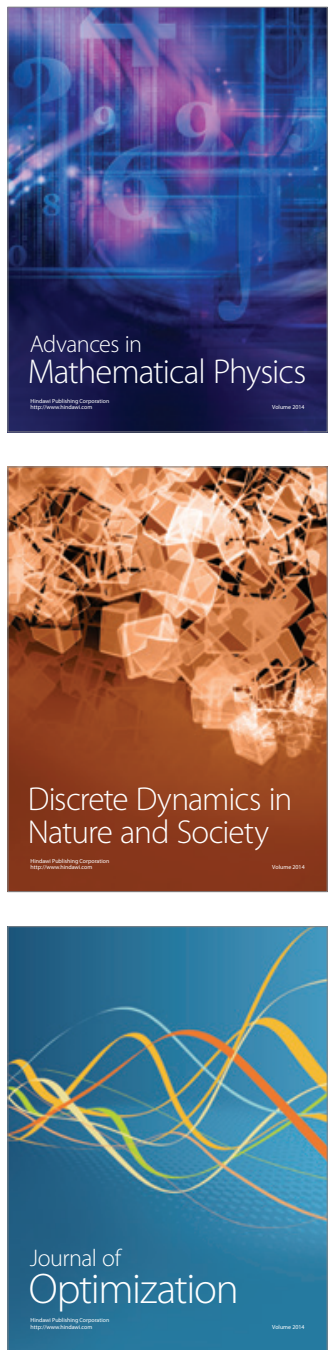